\title{
A GRID-BASED BOUNDARY INTEGRAL METHOD FOR ELLIPTIC PROBLEMS IN THREE DIMENSIONS*
}

\author{
J. THOMAS BEALE ${ }^{\dagger}$
}

\begin{abstract}
We develop a simple, efficient numerical method of boundary integral type for solving an elliptic partial differential equation in a three-dimensional region using the classical formulation of potential theory. Accurate values can be found near the boundary using special corrections to a standard quadrature. We treat the Dirichlet problem for a harmonic function with a prescribed boundary value in a bounded three-dimensional region with a smooth boundary. The solution is a double layer potential, whose strength is found by solving an integral equation of the second kind. The boundary surface is represented by rectangular grids in overlapping coordinate systems, with the boundary value known at the grid points. A discrete form of the integral equation is solved using a regularized form of the kernel. It is proved that the discrete solution converges to the exact solution with accuracy $O\left(h^{p}\right), p<5$, depending on the smoothing parameter. Once the dipole strength is found, the harmonic function can be computed from the double layer potential. For points close to the boundary, the integral is nearly singular, and accurate computation is not routine. We calculate the integral by summing over the boundary grid points and then adding corrections for the smoothing and discretization errors using formulas derived here; they are similar to those in the two-dimensional case given by [J. T. Beale and M.-C. Lai, SIAM J. Numer. Anal., 38 (2001), pp. 1902-1925]. The resulting values of the solution are uniformly of $O\left(h^{p}\right)$ accuracy, $p<3$. With a total of $N$ points, the calculation could be done in essentially $O(N)$ operations if a rapid summation method is used.
\end{abstract}

Key words. boundary integral methods, nearly singular integrals, potential theory, Dirichlet problem, overlapping grids

AMS subject classifications. 65R20, 65D30, 31B10, 35J25

DOI. $10.1137 / \mathrm{S} 0036142903420959$

1. Introduction. In this paper we develop a simple, direct numerical method of boundary integral type for the solution of an elliptic boundary value problem in a three-dimensional (3D) region with a smooth boundary. We emphasize the Dirichlet problem for an interior harmonic function, although a larger class of problems can be treated. We assume the boundary surface is represented by several overlapping grids, each rectangular in some coordinate system. Values of the solution are to be found at arbitrary points in space (typically, those on a regular 3D grid), some of which will be close to the surface. We do not assume any relationship between the coordinate grids on the surface and the interior grid. We use the classical formulation of the Dirichlet problem in potential theory: The solution can be written as a double layer potential, or dipole layer, with an unknown strength. This dipole strength is determined by a Fredholm integral equation of the second kind on the surface. We solve a discretized form of this integral equation at the coordinate grid points on the surface, replacing the Green's function in the layer potential with a regularized version and the integral with a sum over grid points. We prove that the solution of the numerical integral equation converges to the exact solution with order $h^{p}$, for any $p<5$, depending on the choice of the smoothing parameter relative to $h$, and that it can be found by a simple iteration. Once the dipole strength is found, the value of the harmonic function at any point is given by the double layer potential. For points away from

${ }^{*}$ Received by the editors January 2, 2003; accepted for publication (in revised form) October 13, 2003; published electronically April 14, 2004. This research was supported by the NSF under grant DMS-0102356.

http://www.siam.org/journals/sinum/42-2/42095.html

${ }^{\dagger}$ Department of Mathematics, Duke University, Durham, NC 27708-0320 (beale@math.duke.edu). 
the boundary, the surface integral can be computed in a standard way, but, at points near the boundary, the integral is nearly singular, and accurate computation is not routine. We find the integral in this case by starting with a standard quadrature, again regularized, and then adding corrections. These corrections account for the errors introduced by the smoothing, or regularization, and by discretization. They are derived by local analysis near the singularity, extending the treatment for a twodimensional (2D) region given in [3]. With grid spacing $h$ on the boundary, the resulting values are accurate to $O\left(h^{p}\right)$ for $p<3$, again depending on smoothing.

Boundary integral formulations of the Laplace or Helmholtz equation in three dimensions are widely used in engineering, especially electromagnetics. They are most often solved numerically by the boundary element method; the surface is triangulated, and the equation holds at collocation points (see, e.g., $[2,15]$ ). The integration of the kernel times a basis function at a point in its support often uses a change of variables or a product integration rule. Special care is also needed for points near the support. High order accuracy can be achieved [2, section 9.2], and the method can be accurate for piecewise smooth surfaces. More direct quadrature, or Nyström, methods have not been widely used. Such a method for polyhedral surfaces, summing over centroids in a triangulation, was shown to converge by Rathsfeld [23]. In [24] he showed that a simple quadrature over a triangulation of a smooth surface gives almost $O\left(h^{2}\right)$ convergence with singularity subtraction, and this can be improved to higher order using product integration. Recently, practical methods of this type have been developed. Canino et al. [8] use a triangulation with quadrature based on a local correction method of Strain, an alternative to product integration. Bruno and Kunyansky [7] use overlapping grids and a partition of unity, as in the present work, but they integrate the singularity by a change of variables. Other current approaches include use of wavelets (see, e.g., [19]) or spherical polynomials (see, e.g., [13]). The difficulty of calculating nearly singular integrals for the field at points near the boundary is well recognized [2, section 7.2.1], but very few works have treated it; two recent approaches are given in [17, 25].

While there are many choices of numerical methods for elliptic problems, with boundary integrals or otherwise, the present approach has several advantages for some applications. The data structure needed to represent the surface is minimal, and calculations on the surface are done in the rectangular grids, with the dependence on the surface reduced to coordinate functions. The integration requires no extra work at the singularity while solving the integral equation. Finally, values of the field near the surface can be found by direct integration, adding the corrections presented here. These attributes may be especially important for a time-dependent calculation with a moving boundary. For viscous, incompressible fluid flow, pressure terms due to a boundary could be found as nearly singular integrals [3]. The linear system resulting from the integral equation is well conditioned. With $n$ boundary points, the direct solution of the linear system would require about $O\left(n^{2}\right)$ operations, but this could be reduced to about $O(n)$ using a rapid summation method such as the fast multipole method of Greengard and Rokhlin [14]. Similarly, fast summation could be used to produce the values of the harmonic function. Another way to produce the interior solution, as observed by Mayo [21] (see also [1]), is to find values near the boundary, compute a discrete Laplacian, extend it by zero to a computational box including the original region, and then invert using a fast Poisson solver to produce values at grid points. This approach was used in the $2 \mathrm{D}$ case in [3, section 4]. Either way, the operation count with a total of $N$ points could be reduced to about $O(N)$. We assume here that the surface and functions are smooth, i.e., have several derivatives 
to justify various Taylor expansions; this method would not be valid for a boundary with corners and edges without further modification.

We now summarize the method and results. We consider the Dirichlet problem for a bounded domain $\Omega \subseteq \mathbf{R}^{3}$ with smooth boundary $\mathcal{S}$. Given a function $g$ on $\mathcal{S}$ we want to find a function $u$ on $\Omega$ such that

$$
\Delta u=0 \quad \text { on } \Omega, \quad u=g \quad \text { on } \mathcal{S} .
$$

The solution can be written as a double layer potential

$$
u(y)=\int_{\mathcal{S}} \frac{\partial}{\partial n(x)} G(x-y) f(x) d S(x)
$$

for some dipole strength $f$, determined by $g$, where $n(x)$ is the unit outward normal at $x \in \mathcal{S}, G$ is the Green's function for $\Delta$ in $\mathbf{R}^{3}, G(x)=-1 / 4 \pi|x|$, and

$$
\frac{\partial}{\partial n(x)} G(x-y)=n(x) \cdot \nabla G(x-y)=\frac{n(x) \cdot(x-y)}{4 \pi|x-y|^{3}} .
$$

The unknown $f$ is the solution of the integral equation on the surface $\mathcal{S}$ :

$$
\frac{1}{2} f(x)+\int_{\mathcal{S}} K\left(x, x^{\prime}\right) f\left(x^{\prime}\right) d S\left(x^{\prime}\right)=g(x), \quad K\left(x, x^{\prime}\right)=\frac{\partial}{\partial n(x)} G\left(x^{\prime}-x\right),
$$

or $f+2 T f=2 g$, where $T$ is the integral operator with kernel $K$. It is known (see, e.g., $\left[18\right.$, section 10.5]) that the iterates $f^{n}$ defined by

$$
f^{n+1}=(1-\beta) f^{n}-2 \beta T f^{n}+2 \beta g
$$

converge to the solution of (1.4), provided $0<\beta<1$.

To describe the numerical formulation of the problem (1.4) for $f$, we suppose the surface $\mathcal{S}$ is covered by several coordinate patches $X^{\sigma}: U_{\sigma} \rightarrow \mathcal{S}$, where $U_{\sigma}$ is an open subset of $\mathbf{R}^{2}$. We assume each $X^{\sigma}: U_{\sigma} \rightarrow \mathbf{R}^{3}$ is smooth and nondegenerate; i.e., $\partial X^{\sigma}(\alpha) / \partial \alpha$ has rank 2 at each point, with $\alpha=\left(\alpha_{1}, \alpha_{2}\right)$. In order to write the integral as a sum of integrals in coordinates, we introduce a partition of unity, that is, a collection of smooth functions $\left\{\psi^{\sigma}\right\}: \mathcal{S} \rightarrow \mathbf{R}$ such that $\psi^{\sigma}$ is zero outside a compact subset of $U_{\sigma}$, called the support of $\psi^{\sigma}$, and $\sum_{\sigma} \psi^{\sigma}(x)=1$ for each $x \in \mathcal{S}$. (See section 5 for typical choices.) We can now write the integral of a function $F$ on $\mathcal{S}$ as

$$
\int_{\mathcal{S}} F\left(x^{\prime}\right) d S\left(x^{\prime}\right)=\sum_{\sigma} \int_{U_{\sigma}} F\left(X^{\sigma}(\alpha)\right) \psi^{\sigma}\left(X^{\sigma}(\alpha)\right) A^{\sigma}(\alpha) d \alpha,
$$

where $A^{\sigma}(\alpha) d \alpha$ is the element of surface area in the $\sigma$ th patch. In solving (1.4) it is helpful to use the familiar identity

$$
\int_{\mathcal{S}} K\left(x, x^{\prime}\right) d S\left(x^{\prime}\right)=\frac{1}{2}
$$

to reduce the order of the singularity; we rewrite (1.4) as

$$
f(x)+\int_{\mathcal{S}} K\left(x, x^{\prime}\right)\left[f\left(x^{\prime}\right)-f(x)\right] d S\left(x^{\prime}\right)=g, \quad x \in S .
$$


Since $K=O(1 / r)$ on $\mathcal{S}$, the resulting integrand is bounded, although not smooth. We will write the integral in (1.8) as in (1.6), replacing $K$ by a regularized version $K_{\delta}$, with a shape factor $s$ and a smoothing parameter $\delta$ to be chosen:

$$
K_{\delta}\left(x, x^{\prime}\right)=n\left(x^{\prime}\right) \cdot \nabla G_{\delta}\left(x^{\prime}-x\right), \quad \nabla G_{\delta}\left(x^{\prime}-x\right)=\nabla G\left(x^{\prime}-x\right) s\left(\left|x-x^{\prime}\right| / \delta\right) .
$$

With the natural choice $G_{\delta}(x)=G(x) \operatorname{erf}(|x| / \delta)=-\operatorname{erf}(|x| / \delta) / 4 \pi|x|$, we have

$$
s(r)=\operatorname{erf}(r)-(2 / \sqrt{\pi}) r e^{-r^{2}},
$$

where erf is the usual error function

$$
\operatorname{erf}(r)=\frac{2}{\sqrt{\pi}} \int_{0}^{r} e^{-t^{2}} d t
$$

In section 2 we find that this choice of smoothing leads to an $O\left(\delta^{3}\right)$ error to the integral in (1.8). Moreover, this can be improved to $O\left(\delta^{5}\right)$ with a slight change to

$$
s(r)=\operatorname{erf}(r)-(2 / \sqrt{\pi})\left(r-2 r^{3} / 3\right) e^{-r^{2}} .
$$

We can now give the discrete form of (1.8). Suppose a grid spacing $h$ is chosen for the coordinate patches, and the $\sigma$ th patch has grid points $x_{i}^{\sigma}=X^{\sigma}(i h)$ for $i \in Z^{2}$ such that $x_{i}^{\sigma} \in V_{\sigma}$, where $V_{\sigma}$ is the interior of the support of $\psi^{\sigma}$. We assume the specified function $g$ is known at these points, say, $g_{i}^{\sigma}=g\left(x_{i}^{\sigma}\right)$. Then

$$
f_{i}^{\sigma}+\sum_{j, \tau} K_{i j}^{\sigma \tau} \psi_{j}^{\tau}\left[f_{j}^{\tau}-f_{i}^{\sigma}\right] A_{j}^{\tau} h^{2}=g_{i}^{\sigma}
$$

is the discrete analogue of (1.8), where

$$
K_{i j}^{\sigma \tau}=K_{\delta}\left(x_{i}^{\sigma}, x_{j}^{\tau}\right), \quad \psi_{j}^{\tau}=\psi^{\tau}\left(x_{j}^{\tau}\right), \quad A_{j}^{\tau}=A^{\tau}(j h) .
$$

We choose $\delta$ in terms of $h$ so that $\delta \rightarrow 0$ as $h \rightarrow 0$, but, for some constant $\rho_{0}$,

$$
\rho=\delta / h \geq \rho_{0}>0 .
$$

We can find the solution $f_{i}^{\sigma}$ as the limit of the iteration corresponding to (1.5),

$$
f_{i}^{\sigma, n+1}=(1-2 \beta) f_{i}^{\sigma, n}-2 \beta \sum_{j, \tau} K_{i j}^{\sigma \tau} \psi_{j}^{\tau}\left[f_{j}^{\tau, n}-f_{i}^{\sigma, n}\right] A_{j}^{\tau} h^{2}+2 \beta g_{i}^{\sigma} .
$$

The following theorem assures the validity of this procedure.

TheOREM 1.1. For the interior Dirichlet problem (1.1), with $\mathcal{S}$ and $g$ smooth, suppose that the grid size $h$ and the smoothing radius $\delta$ are chosen sufficiently small subject to the condition (1.15). Then the discrete integral equation (1.13), using the regularization (1.9), (1.12), has a unique solution $f_{i}^{\sigma}$, which converges uniformly to the exact solution $f$ of (1.4) or (1.8) as $h \rightarrow 0, \delta \rightarrow 0$, with the estimate

$$
\left|f_{i}^{\sigma}-f\left(x_{i}^{\sigma}\right)\right| \leq C_{1} \delta^{5}+C_{2} h^{2} e^{-c_{0}(\delta / h)^{2}} .
$$

Here $c_{0}$ depends only on the coordinate patches, but $C_{1}, C_{2}$ depend on bounds for derivatives of $f$. For $0<\beta<1$, the iterates defined by (1.16) converge uniformly to the discrete solution $f_{i}^{\sigma}$, with a rate independent of $h$ and $\delta$. 
The convergence proof must take into account the agreement of function values where the grids overlap. This is done using a discrete Hölder norm. The error from the smoothing gives the first term in (1.17); the discretization contributes the second term, plus a remainder smaller than the first. The second term is dominated by the first if $\rho=\delta / h$ grows slowly as $h, \delta \rightarrow 0$. For example, if we choose $\delta=c h^{q}$, with $q<1$, the error is $O\left(\delta^{5}\right)=O\left(h^{5 q}\right)$. Similarly, if we use the simpler choice of smoothing (1.10) we obtain almost $O\left(h^{3}\right)$ convergence. The constant $c_{0}$ is $\pi^{2} \gamma^{2} / 2$, where $\gamma$, given by (3.19), depends on $g^{i j}$; see (3.25).

After solving the discrete integral equation for the dipole strength $f$ at the coordinate grid points $x_{i}^{\sigma}$, we now want to find the values of the solution $u$ of (1.1) from the double layer representation (1.2). For a point $y$ away from the surface we can discretize the integral routinely, since the integrand is smooth. For $y$ near the surface, however, the integral is nearly singular, and accurate calculation requires more care. In contrast to the case $y \in \mathcal{S}, n(x) \cdot \nabla G(x-y)=O\left(|x-y|^{-2}\right)$. Given $y$ near $\mathcal{S}$, there is a point $x_{0}$ on the surface so that $y$ is on the normal line through $x_{0}$; i.e., $y=x_{0}+b n_{0}$, for some $b$, where $n_{0}$ is the outward unit normal at $x_{0}$. We can use Green's identity to replace (1.2) with a subtracted form

$$
u(y)=\int_{\mathcal{S}} \frac{\partial}{\partial n(x)} G(x-y)\left[f(x)-f\left(x_{0}\right)\right] d S(x)+f\left(x_{0}\right), \quad y \in \Omega .
$$

We can approximate the integral above by the sum

$$
S=\sum_{\sigma, j} n\left(x_{j}^{\sigma}\right) \cdot \nabla G_{\delta}\left(x_{j}^{\sigma}-y\right)\left[f\left(x_{j}^{\sigma}\right)-f\left(x_{0}\right)\right] \psi_{j}^{\sigma} A_{j}^{\sigma} h^{2} .
$$

We use the simpler regularization (1.10); it seems that higher order smoothing cannot be incorporated in the modified kernel for the nearly singular case. This sum typically has a large error for $y$ near $S$. We can think of the error in two parts: the smoothing error from replacing $\nabla G$ by $\nabla G_{\delta}$ and the discretization error from replacing the integral with $\nabla G_{\delta}$ by the sum or, briefly,

$$
\sum_{\delta}-\int=\left(\int_{\delta}-\int\right)+\left(\sum_{\delta}-\int_{\delta}\right) .
$$

The analysis of the following sections shows that these errors, uniform with respect to $y$ near $\mathcal{S}$, are $O\left(\delta^{2}\right)$ and $O(h)$, respectively. However, the largest errors can be removed by corrections which we now describe. They are derived in sections 2 and 3 and are analogous to those found in the $2 \mathrm{D}$ case in [3].

The corrections involve the geometry of the surface near $x_{0}$. There is at least one $\sigma$ so that $x_{0}$ is in the $\sigma$ th patch; i.e., $x_{0}=X^{\sigma}\left(\alpha_{0}\right)$ for some $\alpha_{0} \in U^{\sigma}$. Let $T_{i}$ be the tangent vector $\left(\partial X^{\sigma} / \partial \alpha_{i}\right)\left(\alpha_{0}\right)$ at $x_{0}, i=1,2$. We use the metric tensor $g_{i j}=T_{i} \cdot T_{j}$; its determinant $g=\operatorname{det}\left(g_{i j}\right)$; the inverse $g^{i j}=\left(g_{i j}\right)^{-1}$; and the surface Laplacian

$$
\Delta f=\sum_{i, j=1}^{2} \frac{1}{\sqrt{g}} \frac{\partial}{\partial \alpha_{j}}\left(\sqrt{g} g^{i j} \frac{\partial\left(f \circ X^{\sigma}\right)}{\partial \alpha_{i}}\right) .
$$

If the grid points $\left\{x_{j}^{\sigma}\right\}$ and values $f_{j}^{\sigma}$ are known, these quantities can be found at $\alpha_{0}$ by interpolation. The smoothing correction derived in section 2 is, with $\lambda=b / \delta$,

$$
\mathcal{T}_{1}=\delta^{2}\left(\Delta f\left(x_{0}\right)\right)(\lambda / 4)\left(|\lambda| \operatorname{erfc}|\lambda|-e^{-\lambda^{2}} / \sqrt{\pi}\right) .
$$


The discretization correction comes from the Poisson summation formula applied to the regularized kernel. It uses the function

$$
E(p, q)=e^{2 p q} \operatorname{erfc}(p+q)+e^{-2 p q} \operatorname{erfc}(-p+q),
$$

where erfc $=1-$ erf is the complementary error function. The point $x_{0}$ may be in more than one coordinate patch, and a correction term is needed for each. In the $\sigma$ th patch, $x_{0}=X^{\sigma}\left(\alpha_{0}\right)$ for some $\alpha_{0}$ depending on $\sigma$. We can write $\alpha_{0}=i h+\nu h$ for some $i \in Z^{2}$ and $\nu=\left(\nu_{1}, \nu_{2}\right)$, with $0 \leq \nu_{s} \leq 1, s=1,2$. The correction for the $\sigma$ th patch is

$$
\begin{gathered}
\mathcal{T}_{2}^{\sigma}=-h \sum_{r=1}^{2} c_{r} \psi^{\sigma}\left(\alpha_{0}\right) \frac{\partial\left(f \circ X^{\sigma}\right)}{\partial \alpha_{r}}\left(\alpha_{0}\right), \\
c_{r}=\frac{\rho \lambda}{2} \sum_{s=1}^{2} \sum_{n \in Q} a(n, s) \sin (2 \pi n \cdot \nu) \frac{g^{r s} n_{s}}{\|n\|} E(\lambda, \pi \rho\|n\|) .
\end{gathered}
$$

Here $Q=\left\{n=\left(n_{1}, n_{2}\right) \in \mathbf{Z}^{2}: n_{2} \geq 0, n \neq 0\right\} ;\|n\|=\sqrt{g^{i j} n_{i} n_{j}} ; a(n, s)=1 / 2$ when $s=1$ and $n_{2}=0$, and $a(n, s)=1$ otherwise.

The following theorem summarizes the error estimates for the computed value of $u(y)$ in (1.2), starting with the sum (1.19) and adding corrections.

TheOrem 1.2. For $y \in \Omega$, let $u(y)$ be the exact solution of (1.1), given by (1.2), assuming $f$ and $\mathcal{S}$ are smooth. Let $\tilde{u}(y)$ be the value computed as the sum

$$
\tilde{u}(y)=S+f\left(x_{0}\right)+\mathcal{T}_{1}+\sum_{\sigma} \mathcal{T}_{2}^{\sigma}
$$

with $S, \mathcal{T}_{1}, \mathcal{T}_{2}^{\sigma}$ given by (1.19), (1.10), (1.21)-(1.25), subject to (1.15). Then the error has the form $\tilde{u}(y)-u(y)=\varepsilon_{1}+\varepsilon_{2}$, where the smoothing error $\varepsilon_{1}$ and the discretization error $\varepsilon_{2}$ can be estimated as follows, uniformly for $y$ near $\mathcal{S}$, with $c_{0}$ as before:

$$
\left|\varepsilon_{1}\right| \leq C_{1} \delta^{3}, \quad\left|\varepsilon_{2}\right| \leq C_{2} h^{2} e^{-c_{0}(\delta / h)^{2}}+C_{3} h^{3} .
$$

Again we can choose $\delta$ so that $\rho=\delta / h$ grows slowly and the error is almost $O\left(h^{3}\right)$. Thus, for $\delta=c h^{q}$ with $q<1$, the error is $O\left(\delta^{3}\right)=O\left(h^{3 q}\right)$. The sum in (1.25) is infinite, but the terms decay at a Gaussian rate, independent of $y$, and only a few terms are needed; see Lemma 3.3. It decreases rapidly as $\rho$ is increased, as does the main term in the estimate for $\varepsilon_{2}$; see (3.20).

Related problems for harmonic functions can be treated; correction formulas for the single layer potential in the nearly singular case will be given elsewhere. Similar methods should apply to the Helmholtz equation since the leading singularities are the same. This approach in two dimensions [3] was applied to the Stokes equations of viscous fluid flow by Cortez [10], and 3D applications should be possible as well. Regularized kernels have long been used for fluid flow in vorticity formulation (see, e.g., $[16,6])$, as well as other physical contexts, and discretization corrections of the present type have also been used in fluid problems [20, 22, 4].

In section 2 we analyze the smoothing error, derive the correction (1.22), and show that (1.12) has $O\left(\delta^{5}\right)$ error on the surface. We begin section 3 with a general lemma about the quadrature of nearly singular integrals with a homogeneous kernel. This is used to derive the correction (1.24), (1.25) and the estimates for the remaining discretization error. Sections 2 and 3 together prove Theorem 1.2 and the consistency part of Theorem 1.1. The rest of the proof of Theorem 1.1 is given in section 4 . In section 5 we present numerical examples, with $\mathcal{S}$ a sphere or ellipsoid, illustrating the general principles. 
2. The smoothing error. In this section we find the first correction to the error in the double layer potential, evaluated at a point near the surface, resulting from the smoothing in the simplest regularized Green's function

$$
G_{\delta}(x)=-\frac{1}{4 \pi} \frac{\operatorname{erf}(r / \delta)}{r}=G(x) \operatorname{erf}(r / \delta), \quad r=|x| .
$$

The correction is $O\left(\delta^{2}\right)$ and leaves an error of $O\left(\delta^{3}\right)$. We also derive the fifth order kernel (1.9), (1.12) for points on the surface.

The smoothing error for a point $y$ near the surface is localized, and we can assume the function $f$ is zero outside one coordinate patch. We can write the error as an integral in this patch, regarding $f$ as a function of $\alpha$,

$$
\varepsilon=\int\left[\nabla G_{\delta}(x(\alpha)-y)-\nabla G(x(\alpha)-y)\right] \cdot n(\alpha) f(\alpha) d S(\alpha) .
$$

For simplicity, we will assume that $x(0)=0$ and $y$ is along the normal line from $x(0)$ so that $y=b n_{0}$ for some $b$, where $n_{0}$ is the unit normal at $x(0)$. Since we always subtract out the principal singularity, we also assume $f(0)=0$. Now

$$
\begin{gathered}
\frac{\partial}{\partial r}\left(G_{\delta}-G\right)=\frac{1}{4 \pi r^{2}} \phi(r / \delta), \\
\phi(\rho)=-\operatorname{erfc}(\rho)+\rho \operatorname{erfc}^{\prime}(\rho)=-\operatorname{erfc}(\rho)-(2 / \sqrt{\pi}) \rho e^{-\rho^{2}} .
\end{gathered}
$$

Thus, with $r=|x(\alpha)-y|$,

$$
\varepsilon=\frac{1}{4 \pi} \int \frac{(x(\alpha)-y) \cdot n(\alpha)}{r^{3}} \phi(r / \delta) f(\alpha) d S(\alpha) .
$$

We will find the largest contribution to $\varepsilon$ using Taylor expansions at $\alpha=0$. To simplify the calculations we first assume the $\alpha$-coordinate system is specially chosen and then extend the result to a general system. With $T_{j}=\partial x / \partial \alpha_{j}$ the tangent vectors to surface, $j=1,2$, we assume that the metric tensor $g_{i j}=T_{i} \cdot T_{j}$ is the identity at $\alpha=0$ and, furthermore, that $\partial g_{i j} / \partial \alpha_{k}=0$ at $\alpha=0, i, j, k=1,2$. The latter is equivalent to assuming that the Christoffel symbols vanish at $\alpha=0$. We also assume, rotating if necessary, that $T_{1}, T_{2}$ have the directions of principal curvature at $\alpha=0$. We then have simple expansions for $x(\alpha)$ and $n(\alpha)$ :

$$
\begin{gathered}
x(\alpha)=T_{1}(0) \alpha_{1}+T_{2}(0) \alpha_{2}+\frac{1}{2} \kappa_{1} n_{0} \alpha_{1}^{2}+\frac{1}{2} \kappa_{2} n_{0} \alpha_{2}^{2}+O\left(|\alpha|^{3}\right), \\
n(\alpha)=n_{0}-\kappa_{1} T_{1}(0) \alpha_{1}-\kappa_{2} T_{2}(0) \alpha_{2}+O\left(|\alpha|^{2}\right),
\end{gathered}
$$

where $\kappa_{1}, \kappa_{2}$ are the principal curvatures. Thus

$$
r^{2}=\left|x-b n_{0}\right|^{2}=|\alpha|^{2}+b^{2}-b \kappa_{1} \alpha_{1}^{2}-b \kappa_{2} \alpha_{2}^{2}+O\left(|\alpha|^{4}\right)+O\left(|\alpha|^{3} b\right) .
$$

We make a further coordinate change $\alpha \mapsto \xi$ to simplify the dependence of $r$ in the integral. We define $\xi_{1}, \xi_{2}$ by requiring $\xi_{j} /|\xi|=\alpha_{j} /|\alpha|$ and $|\xi|^{2}+b^{2}=r^{2}$, or

$$
|\xi|^{2}=|\alpha|^{2}-b \kappa_{1} \alpha_{1}^{2}-b \kappa_{2} \alpha_{2}^{2}+O\left(|\alpha|^{4}\right)+O\left(|\alpha|^{3} b\right)
$$

so that

$$
|\xi|=|\alpha|\left(1-\frac{1}{2} b \kappa_{1} \frac{\alpha_{1}^{2}}{|\alpha|^{2}}-\frac{1}{2} b \kappa_{2} \frac{\alpha_{2}^{2}}{|\alpha|^{2}}\right)+O\left(|\alpha|^{3}\right)+O\left(b^{3}\right) .
$$


For $\alpha$ near 0 , we can solve for $|\alpha|$ to get $|\alpha|=|\xi|(1+b q / 2)+O\left(|\xi|^{3}+b^{3}\right)$ and then

$$
\begin{gathered}
\alpha_{j}=\left(\xi_{j} /|\xi|\right)|\alpha|=(1+b q / 2) \xi_{j}+O\left(|\xi|^{3}\right)+O\left(b^{3}\right), \\
q=\kappa_{1} \xi_{1}^{2} /|\xi|^{2}+\kappa_{2} \xi_{2}^{2} /|\xi|^{2} .
\end{gathered}
$$

We can now write the smoothing error in the form

$$
\varepsilon=\frac{1}{4 \pi} \int \frac{\phi\left(\sqrt{|\xi|^{2}+b^{2}} / \delta\right)}{\left(|\xi|^{2}+b^{2}\right)^{3 / 2}} w(\xi, b) d \xi
$$

with the nonradial factors combined into

$$
w(\xi, b)=[(x-y) \cdot n] f\left|\frac{\partial \alpha}{\partial \xi}\right|\left|T_{1} \times T_{2}\right| .
$$

Next we approximate each of these factors. First, using the expressions above for $x(\alpha)$ and $n(\alpha)$ we find, with $y=b n_{0}$,

$$
(x(\alpha)-y) \cdot n(\alpha)=-b-\frac{1}{2} \kappa_{j} \alpha_{j}^{2}+O\left(|\alpha|^{3}\right)+O\left(b^{3}\right),
$$

summed over $j$, and, since $\alpha_{j}=\xi_{j}+O(b)$,

$$
(x-y) \cdot n=-b-\frac{1}{2} q|\xi|^{2}+O\left(|\xi|^{3}\right)+O\left(b^{3}\right) .
$$

Next, with $\alpha$-derivatives of $f$ at $\alpha=0$ denoted by $f_{i}$ or $f_{i j}$, we have

$$
f=f_{j} \alpha_{j}+\frac{1}{2} f_{i j} \alpha_{i} \alpha_{j}+O\left(|\alpha|^{3}\right),
$$

since $f(0)=0$, with sums over $i$ and $j$, or

$$
f=f_{j}\left(1+\frac{b q}{2}\right) \xi_{j}+\frac{1}{2} f_{i j} \xi_{i} \xi_{j}+O\left(|\xi|^{3}\right)+O\left(b^{3}\right) .
$$

For the Jacobian, since $q$ depends only on $\xi /|\xi|$, we find from (2.10) that

$$
\operatorname{det}(\partial \alpha / \partial \xi)=1+b q+O\left(|\xi|^{2}\right)+O\left(b^{2}\right) .
$$

Finally, from (2.6) we have $T_{j}(\alpha)=T_{j}(0)+\kappa_{j} \alpha_{j} n_{0}$, and, since $T_{j}(0) \perp n(0)$,

$$
\left|T_{1} \times T_{2}\right|=1+O\left(|\alpha|^{2}\right)=1+O\left(|\xi|^{2}\right) .
$$

To approximate (2.12), we now substitute (2.14)-(2.17) into (2.13) and obtain

$$
w(\xi, b)=-b \xi_{j} f_{j}-(3 q / 2) b^{2} \xi_{j} f_{j}-(q / 2)|\xi|^{2} \xi_{j} f_{j}-(b / 2) f_{i j} \xi_{i} \xi_{j}+R(\xi, b),
$$

where $R=O\left(|\xi|^{4}+b^{4}\right)$. The first three terms are odd in $\xi$ and will contribute zero to the integral (2.12). We check that the remainder $R$ is negligible: With the change of variables $\xi=\delta \zeta, b=\delta \lambda$, we can write $R(\xi, b)=\delta^{4} \tilde{R}(\zeta, \lambda)$ for some bounded function $\tilde{R}$. Then the contribution to (2.12) from the remainder $R$ in $w$ is

$$
(4 \pi)^{-1} \delta^{-3+4+2} \int \frac{\phi\left(\sqrt{|\zeta|^{2}+\lambda^{2}}\right)}{\left(|\zeta|^{2}+\lambda^{2}\right)^{3 / 2}} \tilde{R}(\zeta, \lambda) d \xi=O\left(\delta^{3}\right) .
$$


We are now left with only the fourth term in (2.18). Because of symmetry, only the terms with $i=j$ contribute, and the integral with $\xi_{j}^{2}$ reduces to a radial one. Again with $\xi=\delta \zeta, b=\delta \lambda$, and $s=|\zeta|,(2.12)$ is now

$$
\varepsilon=-\delta^{2} \frac{\lambda}{8}\left(f_{11}+f_{22}\right) \int_{0}^{\infty} \frac{\phi\left(\sqrt{s^{2}+\lambda^{2}}\right)}{\left(s^{2}+\lambda^{2}\right)^{3 / 2}} s^{3} d s+O\left(\delta^{3}\right) .
$$

To evaluate the integral, let

$$
I(\lambda)=\int_{0}^{\infty}\left(G_{1}(r)-G(r)\right) s^{3} d s, \quad r^{2}=s^{2}+\lambda^{2}
$$

then $d I / d \lambda$ is similar to the above:

$$
I^{\prime}(\lambda)=\frac{\lambda}{4 \pi} \int_{0}^{\infty} \frac{\phi(r)}{4 \pi r^{3}} s^{3} d s
$$

Changing the variable of integration to $r$, we have

$$
\begin{gathered}
I(\lambda)=\int_{|\lambda|}^{\infty}\left(G_{1}(r)-G(r)\right) s^{2} r d r=(4 \pi)^{-1} \int_{|\lambda|}^{\infty} \operatorname{erfc}(r)\left(r^{2}-\lambda^{2}\right) d r, \\
I^{\prime}(\lambda)=-(2 \pi)^{-1} \lambda \int_{|\lambda|}^{\infty} \operatorname{erfc}(r) d r=(2 \pi)^{-1} \lambda\left(|\lambda| \operatorname{erfc}|\lambda|-e^{-\lambda^{2}} / \sqrt{\pi}\right),
\end{gathered}
$$

and, finally,

$$
\varepsilon=-\delta^{2}\left(f_{11}+f_{22}\right)(\lambda / 4)\left(|\lambda| \operatorname{erfc}|\lambda|-e^{-\lambda^{2}} / \sqrt{\pi}\right)+O\left(\delta^{3}\right) .
$$

We have now obtained the smoothing correction with derivatives expressed in the special coordinates. To restate the answer in a general coordinate system, we have only to note the invariant form of the Laplacian on the surface, given by (1.21). It reduces to $f_{11}+f_{22}$ at $\alpha=0$ in the special case above. Therefore it is also the correct expression in arbitrary coordinates. Thus $\varepsilon=-\mathcal{T}_{1}+O\left(\delta^{3}\right)$, with $\mathcal{T}_{1}$ given by (1.22), justifying the stated correction and smoothing error.

The fifth order kernel. In view of the above analysis, we can identify the source of the $O\left(\delta^{3}\right)$ error in the special case when the point $y$ is on the surface. We can then modify the choice of $G_{\delta}$ to remove this error, resulting in smoothing that is $O\left(\delta^{5}\right)$ accurate on the surface. With $y$ on the surface, the smoothing error $\varepsilon$ is given by (2.12) with $b=0$. From (2.18) we see that the terms in the expansion of $w(\xi, 0)$ up to order 3 make no contribution. The fourth order terms will give an error proportional to

$$
\int \phi(|\xi| / \delta)|\xi|^{-3}|\xi|^{4} d \xi=\delta^{3} \int \phi(|\zeta|)|\zeta| d \zeta=2 \pi \delta^{3} \int_{0}^{\infty} \phi(r) r^{2} d r .
$$

The fifth order terms will lead to odd integrands, and thus the remaining error is $O\left(\delta^{5}\right)$. To eliminate the $O\left(\delta^{3}\right)$ error, we simply have to change the choice of $G_{\delta}$, and therefore $\phi$, so that the last integral is zero. Our new choice of $G_{\delta}$ will be so that $\phi(r)$ is replaced by $\phi_{5}(r)=\phi(r)+a r \phi^{\prime}(r)$ with $a$ a constant. It is easy to see from an integration by parts that the integral above is zero, with $\phi_{5}$ in place of $\phi$, provided we take $a=1 / 3$. Thus $\phi_{5}(r)=\operatorname{erf}(r)-1-(2 / \sqrt{\pi})\left(r-2 r^{3} / 3\right) e^{-r^{2}}$, and the modification of $\nabla G$ is $\nabla G_{\delta}(x)=\nabla G(x)\left[1+\phi_{5}(|x| / \delta)\right]$ as given by (1.9), (1.12). 
3. The discretization error. We begin with a general principle, Lemma 3.1, for the quadrature of nearly singular integrals. We apply this to a local approximation of the double layer potential in Lemma 3.3, obtaining the corrections (1.24), (1.25). We then compare with the exact potential, verifying the discretization estimate of Theorem 1.2, and find an improved estimate needed for Theorem 1.1.

Our treatment of the discretization error is based on a lemma describing the quadrature error for the integral of a regularized homogeneous function $K_{\delta}$, times a smooth function $f$, over a plane displaced from the origin. This lemma is similar to Lemma 3.1 of [3], except that we do not assume here that the smoothing radius $\delta$ is proportional to the grid size $h$; we allow $\rho=\delta / h$ unbounded as $h \rightarrow 0$. Let $K$ be a homogeneous function of $(x, y) \in \mathbf{R}^{d} \times \mathbf{R}$ with degree $m$, that is,

$$
K(a x, a y)=a^{m} K(x, y), \quad a>0, \quad(x, y) \neq 0 .
$$

We choose a regularization of the form

$$
K_{\delta}(x, y)=K(x, y) s(x / \delta, y / \delta), \quad(x, y) \in \mathbf{R}^{d} \times \mathbf{R},
$$

where $s$ is a specified shape function such that $s \rightarrow 1$ rapidly at infinity. It follows that $K_{\delta}(x, y)=\delta^{m} K_{1}(x / \delta, y / \delta)$. The lemma concerns the integral of $K_{\delta} f$ over $x$ with $y$ fixed. We allow the singularity to be misaligned from the grid points in $x$-space.

LEMma 3.1. Let $K_{\delta}$ be a smooth function on $\mathbf{R}^{d+1}$ with the form (3.2) such that $K$ and $s$ are smooth for $(x, y) \neq 0 ; K$ is homogeneous of degree $m$ with $-d \leq m \leq 0$; $s(x, y) \rightarrow 1$ as $(x, y) \rightarrow \infty$; and $\left|D^{\beta} s(x, y)\right| \leq C_{\beta}|(x, y)|^{-|\beta|}$ for $|(x, y)| \geq 1$ and for each multi-index $\beta$. Let $f$ be a smooth function on $\mathbf{R}^{d}$ such that $f$ and its derivatives are rapidly decreasing. Suppose, for each $h>0$, that $\delta$ is chosen so that $\rho=\delta / h \geq \rho_{0}$ for some fixed $\rho_{0}>0$. Assume $\nu \in \mathbf{R}^{d}$ with $0 \leq \nu_{j} \leq 1$ for $1 \leq j \leq d$. Let $\eta=y / h$. Then the error in replacing the integral I by the sum $S$,

$$
\begin{gathered}
I=\int_{\mathbf{R}^{d}} K_{\delta}(x, y) f(x) d x=\int_{\mathbf{R}^{d}} K(x, y) s(x / \delta, y / \delta) f(x) d x, \\
S=\sum_{n \in \mathbf{Z}^{d}} K_{\delta}(n h-\nu h, y) f(n h-\nu h) h^{d},
\end{gathered}
$$

has the form

$$
\begin{gathered}
S-I=h^{d+m}\left(c_{0} f(0)+C_{1} h+C_{2} h^{2}+\cdots+C_{\ell} h^{\ell}\right)+O\left(\delta^{d+m+\ell+1}\right), \\
c_{0}=(2 \pi)^{d / 2} \sum_{0 \neq n \in \mathbf{Z}^{d}} e^{-2 \pi i n \nu} \hat{K}_{\rho}(2 \pi n, \eta) .
\end{gathered}
$$

Here $\hat{K}_{\rho}(\cdot, \eta)$ is the Fourier transform of $K_{\rho}(\cdot, \eta)$, and $\ell$ depends on the smoothness of $K, s, f$. The leading constant $c_{0}$ is uniformly bounded in $\eta, \rho, \nu$. The $C_{j}$, for $j \geq 1$, and the error term are uniformly bounded in $\eta, \rho, \nu$, depending on $f$.

The value of $c_{0}$ is derived from the Poisson summation formula applied to $K_{\rho}$. The sum (3.6) converges rapidly. We write the Fourier transform as

$$
\hat{F}(k)=(2 \pi)^{-d / 2} \int F(x) e^{-i k x} d x .
$$

The proof is a modification of that for Lemma 3.1 in [3] and related to ones in $[4,12]$.

When we apply this lemma to the double layer potential, evaluated at a point $y$ near the surface, the leading error comes from the part of the surface close to $y$, 
and we begin with a simplified approximation for the potential. Suppose $y=b n_{0}$, where $n_{0}$ is the unit normal to a point $x\left(\alpha_{0}\right)$ on the surface. For convenience, we take $\alpha_{0}=0$ and $x\left(\alpha_{0}\right)=0$. With $T_{j}, g_{i j}, g^{i j}$ as defined earlier, at $\alpha=0$, let $\tau=\left|T_{1} \times T_{2}\right|$ so that $\operatorname{det} g_{i j}=\tau^{2}$. Also let $J=\partial x(0) / \partial \alpha$ so that $J \alpha=T_{1} \alpha_{1}+T_{2} \alpha_{2}$. For $\alpha$ near 0 , we can approximate $x(\alpha)$ by its projection $J \alpha$ in the tangent plane and the double layer kernel $n(\alpha) \cdot \nabla G_{\delta}(x(\alpha), y)$ by

$$
K_{\delta}^{(0)}(\alpha, b)=n_{0} \cdot \nabla G_{\delta}\left(J \alpha-b n_{0}\right)=-(\partial / \partial b) G_{\delta}\left(J \alpha-b n_{0}\right) .
$$

The surface area $d S(\alpha)$ is $\tau d \alpha$ to first approximation. We subtract out the singularity in the double layer potential, and the leading contribution to $f(\alpha)-f(0)$ will be $\left(\alpha_{1} \partial_{1} f+\alpha_{2} \partial_{2} f\right) \zeta(\alpha)$ with $\partial_{r} f=\partial_{r} f(0)$ for some cut-off function $\zeta, \zeta=1$ near $\alpha=0$. Thus we actually apply the lemma to the kernel

$$
K_{\delta}^{(r)}(\alpha, b)=K_{\delta}^{(0)}(\alpha, b) \alpha_{r}, \quad r=1,2 .
$$

We first show that this simplified case gives the $O(h)$ error stated in (1.24), (1.25).

Lemma 3.2. With the notation above, let

$$
I=\int_{R^{2}} n_{0} \cdot \nabla G_{\delta}\left(J \alpha-b n_{0}\right)\left(\alpha_{1} \partial_{1} f+\alpha_{2} \partial_{2} f\right) \zeta(\alpha) \tau d \alpha
$$

and $S$ be the corresponding sum with $\alpha=j h-\nu h$. Then $S-I=\left(c_{1} \partial_{1} f+c_{2} \partial_{2} f\right) h+$ $O\left(h^{p}\right)$ for large $p$, where $c_{1}, c_{2}$ are given by (1.25).

Proof. Our main task is to find the Fourier transform of $K_{\rho}^{(r)}(\alpha, b)$ in $\alpha$ alone. We begin with the 3D Fourier transform of $G_{\rho}(x)=-\operatorname{erf}(|x| / \rho) / 4 \pi|x|$,

$$
G_{\rho}^{\wedge}(k)=-(2 \pi)^{-3 / 2}|k|^{-2} e^{-\rho^{2}|k|^{2} / 4}, \quad k=\left(k_{1}, k_{2}, k_{3}\right) \in \mathbf{R}^{3} .
$$

We interpret $G_{\rho}\left(J \alpha-b n_{0}\right)$ as a composition: Since $G_{\rho}$ is radial, it depends on $\alpha$ only through $|J \alpha|^{2}=|B \alpha|^{2}$, where $B=\left(J^{*} J\right)^{1 / 2}$; note $|B \alpha|^{2}=J^{*} J \alpha \cdot \alpha=\sum_{i j} g_{i j} \alpha_{i} \alpha_{j}$. Thus $G_{\rho}\left(J \alpha-b n_{0}\right)=\left(G_{\rho} \circ M\right)(\alpha, b)$, where $M(\alpha, b)=(B \alpha, b)$. The 3D transform of $G_{\rho} \circ M$, as a function of $(\alpha, b)$, is then

$$
\left(G_{\rho} \circ M\right)^{\wedge}(k)=|\operatorname{det} M|^{-1} G_{\rho} \wedge\left(\left(M^{*}\right)^{-1} k\right)=\tau^{-1} G_{\rho}{ }^{\wedge}\left(B^{-1}\left(k_{1}, k_{2}\right), k_{3}\right),
$$

and, since $G_{\rho}{ }^{\wedge}$ is radial and $B^{-2}=\left(J^{*} J\right)^{-1}$,

$$
\left(G_{\rho} \circ M\right)^{\wedge}(k)=\tau^{-1} G_{\rho}^{\wedge}\left(\ell, k_{3}\right), \quad \ell^{2}=\sum_{i, j=1}^{2} g^{i j} k_{i} k_{j} .
$$

Now

$$
\hat{K}_{\rho}^{(0)}(k)=\left(-(\partial / \partial b) G_{\rho} \circ M\right)^{\wedge}(k)=-i k_{3} \tau^{-1} G_{\rho}{ }^{\wedge}\left(\ell, k_{3}\right),
$$

and the transform of $K_{\rho}^{(0)}(\alpha, b)$ in $\alpha$ alone is

$$
\begin{aligned}
K_{\rho}^{(0)}(\cdot, b)^{\wedge}\left(k_{1}, k_{2}\right)=(2 \pi)^{-1 / 2} \int_{-\infty}^{\infty} \hat{K}_{\rho}^{(0)}\left(k_{1}, k_{2}, k_{3}\right) e^{i k_{3} b} d k_{3} \\
=(2 \pi)^{-2} \tau^{-1} \frac{\partial}{\partial b} \int_{-\infty}^{\infty} \frac{1}{\ell^{2}+k_{3}^{2}} e^{-\rho^{2}\left(\ell^{2}+k_{3}^{2}\right) / 4} e^{i k_{3} b} d k_{3} .
\end{aligned}
$$


This is similar to (3.29) in [3]; we find

$$
K_{\rho}^{(0)}(\cdot, b)^{\wedge}\left(k_{1}, k_{2}\right)=(8 \pi \tau)^{-1}\left[e^{\ell b} \operatorname{erfc}(b / \rho+\ell \rho / 2)-e^{-\ell b} \operatorname{erfc}(-b / \rho+\ell \rho / 2)\right] .
$$

Next, since $K_{\rho}^{(r)}=K_{\rho}^{(0)} \alpha_{r}, \hat{K}_{\rho}^{(r)}\left(k_{1}, k_{2}, b\right)$ is given by

$$
\hat{K}_{\rho}^{(r)}=i\left(\partial / \partial k_{r}\right) \hat{K}_{\rho}^{(0)}=i\left(\partial \ell / \partial k_{r}\right)(\partial / \partial \ell) \hat{K}_{\rho}^{(0)}=i\left(g^{r s} k_{s} / \ell\right)(\partial / \partial \ell) \hat{K}_{\rho}^{(0)},
$$

summed over $s$. After differentiating and canceling we get

$$
\hat{K}_{\rho}^{(r)}\left(k_{1}, k_{2}, b\right)=\frac{i b}{8 \pi \tau} \sum_{s=1}^{2} \frac{g^{r s} k_{s}}{\ell} E\left(\frac{b}{\rho}, \frac{\ell \rho}{2}\right)
$$

with $E(p, q)$ given by (1.23). We are now ready to apply Lemma 3.1 to the simplified integral (3.9). The kernel $K_{\rho}^{(r)}(\alpha, b)$ is homogeneous with degree $m=-1$, and, according to the lemma, $S-I=\varepsilon h+O\left(h^{p}\right)$, with

$$
\varepsilon=2 \pi \tau \sum_{r=1}^{2} \sum_{n \neq 0} e^{-2 \pi i n \nu}\left(\partial_{r} f\right) \hat{K}_{\rho}^{(r)}(2 \pi n, b / h),
$$

and, using (3.17), with $\lambda=b / \delta$ and $\|n\|=\sqrt{g^{i j} n_{i} n_{j}}$,

$$
\varepsilon=\frac{i \rho \lambda}{4} \sum_{r, s=1}^{2} \sum_{n \neq 0} e^{-2 \pi i n \nu}\left(\partial_{r} f\right) \frac{g^{r s} n_{s}}{\|n\|} E(\lambda, \pi \rho\|n\|) .
$$

Combining terms for $n$ and $-n$, we find $\varepsilon=c_{1} \partial_{1} f+c_{2} \partial_{2} f$, with $c_{1}, c_{2}$ expressed as in (1.25), and the lemma is proved.

To understand the dependence of the errors on $\rho=\delta / h$, we will use the following lemma concerning the size of the function $E$ of (1.23).

Lemma 3.3. With $E$ as in (1.23), we have for $q \geq q_{0}>0$ and any $p$,

$$
E(p, q) \leq C_{0} \exp \left(-q_{0} p-q^{2}\right)
$$

where $C_{0}$ depends on $q_{0}$.

Proof. Since $E$ is even in $p$ we assume $p \geq 0$. Call the two terms $T_{1}$ and $T_{2}$. Since erfc $a \leq C \exp \left(-a^{2}\right)$ for $a \geq 0, T_{1} \leq C \exp \left(2 p q-(p+q)^{2}\right)=C \exp \left(-p^{2}-\right.$ $\left.q^{2}\right)$. If $q \geq p$, then $T_{2} \leq C \exp \left(-p^{2}-q^{2}\right)$ in the same way. If $q \leq p$, then $T_{2} \leq$ $2 \exp (-2 p q)=2 \exp (-p q) \exp (-p q) \leq 2 \exp (-p q) \exp \left(-q^{2}\right)$. Thus in any case $E \leq$ $\left[C \exp \left(-p^{2}\right)+2 \exp \left(-q_{0} p\right)\right] \exp \left(-q^{2}\right)$, and the result follows from this.

Suppose now that we assume a lower bound for the matrix $g^{i j}$,

$$
\|k\|^{2} \equiv \sum_{i j} g^{i j} k_{i} k_{j} \geq \gamma^{2}|k|^{2}
$$

for some $\gamma>0$. Then applying (3.18) to $\varepsilon$ above, we have $\rho|\lambda| E(\lambda, \pi \rho\|n\|) \leq$ $C \rho \exp \left(-\pi^{2} \rho^{2} \gamma^{2}|n|^{2}\right)$, and, after summing,

$$
\left|c_{r}\right| \leq C \rho \exp \left(-\pi^{2} \rho^{2} \gamma^{2}\right), \quad r=1,2 .
$$


We can now complete the discretization error estimate in Theorem 1.2. With $\alpha=0, x(0)=0$ as before, and assuming $f(0)=0$ for simplicity, we compare

$$
\begin{gathered}
I=\int n(\alpha) \cdot \nabla G_{\delta}(x(\alpha)-y) f(\alpha) d S(\alpha), \\
S=\sum_{j} n\left(\alpha_{j}\right) \cdot \nabla G_{\delta}\left(x\left(\alpha_{j}\right)-y\right) f\left(\alpha_{j}\right) A_{j} h^{2},
\end{gathered}
$$

where $\alpha_{j}=j h-\nu h$ and $A_{j} d \alpha=d S\left(\alpha_{j}\right)$. We will show that, assuming (3.19),

$$
S-I=\left(c_{1} \partial_{1} f+c_{2} \partial_{2} f\right) h+O\left(h^{2} \rho^{3} \exp \left(-\pi^{2} \gamma^{2} \rho^{2}\right)\right)+O\left(h^{3}\right)
$$

with errors uniform in $b, \rho, \nu$. The estimate in (1.27) follows, with $c_{0}=\pi^{2} \gamma^{2} / 2$. We need to show that the error from replacing (3.21) by the simplified version (3.9) is bounded by the remainder in (3.23). This is the quadrature error for the integral of

$$
n(\alpha) \nabla G_{\delta}\left(x(\alpha)-b n_{0}\right) f(\alpha) A(\alpha)-n_{0} \nabla G_{\delta}\left(J \alpha-b n_{0}\right)\left[\alpha_{1} \partial_{1} f(0)+\alpha_{2} \partial_{2} f(0)\right] A(0) .
$$

We can suppose $f$ is zero outside a neighborhood of $\alpha=0$, since the outer part is smooth and gives a high order error. We can add and subtract to write (3.24) as a sum of terms, each a regularized homogeneous function in $(\alpha, b)$ of degree 0 , times a smooth function, as in Lemma 3.1, plus a higher degree remainder. For the remainder we can show the quadrature error is $O\left(h^{3}\right)$ as in the end of the proof of Lemma 3.1 in [3]. The degree 0 terms are similar to the main term treated in Lemma 3.2; they involve $\nabla G_{\delta}$ or $D^{2} G_{\delta}$ and up to two more factors of $\alpha$. By Lemma 3.1, the $O\left(h^{2}\right)$ errors resulting from these terms are given by expressions like those in Lemma 3.2. The Fourier transform of the kernel is very similar to that case, with a second $\alpha$ derivative leading to a factor of $k$ in the transform. An $\alpha$-factor corresponds to a $k$-derivative, leading to an extra factor of $\rho$ in the estimate like (3.20). Otherwise the $O\left(h^{2}\right)$ correction term is bounded as in (3.20), and the estimate (3.23) results.

Finally, we discuss the special case with $y$ on the surface to obtain an improved estimate needed for the discretization error in (1.17) of Theorem 1.1. In this case $b=0$, $\lambda=0$, and the $O(h)$ correction term in (3.23) is zero. The expansion in the proof of (3.23) can be continued further, with successive regularized homogeneous terms of degree $\leq 2$ and an $O\left(h^{5}\right)$ remainder. In this way we find, for $y$ on the surface, $S-I=O\left(h^{2} \rho^{k} \exp \left(-\pi^{2} \gamma^{2} \rho^{2}\right)\right)+O\left(h^{5}\right)$ for some integer $k$. The fifth order kernel used in solving the integral equation has an added term; it has Gaussian form, and the last estimate applies as well with this new term. Finally, since $\rho^{k} \exp \left(-\pi^{2} \gamma^{2} \rho^{2} / 2\right)$ is bounded we can write

$$
S-I=O\left(h^{2} \exp \left(-\pi^{2} \gamma^{2} \rho^{2} / 2\right)\right)+O\left(h^{5}\right) .
$$

This estimate is used in section 4 to prove (1.17) with $c_{0}=\pi^{2} \gamma^{2} / 2$.

4. The integral equation. In this section we prove the assertions about the numerical solution of the integral equation in Theorem 1.1. The exact integral equation, for unknown $f$ on the surface $\mathcal{S}$, with $g$ prescribed on $\mathcal{S}$, has the form

$$
\frac{1}{2} f+T f=g, \quad(T f)(x)=\int_{\mathcal{S}} K\left(x, x^{\prime}\right) f\left(x^{\prime}\right) d S\left(x^{\prime}\right),
$$


where $T$ is the double layer potential, with kernel $K\left(x, x^{\prime}\right)=n\left(x^{\prime}\right) \cdot \nabla G\left(x^{\prime}-x\right)$. We can use the coordinate patches to write the integral operator as

$$
T f(x)=\sum_{\sigma} \int K\left(x, X^{\sigma}(\alpha)\right) \psi^{\sigma}\left(X^{\sigma}(\alpha)\right) f\left(X^{\sigma}(\alpha)\right) A^{\sigma}(\alpha) d \alpha .
$$

Subtracting $f(x)$ inside the integral, we convert (4.1) to the equivalent form (1.8). We are concerned with the discrete version (1.13), (1.14) of (1.8).

We can regard $T$ as a bounded operator on $L^{p}(\mathcal{S})$ for any $p$ with $2 \leq p<\infty$. From potential theory we know that $\frac{1}{2}+T$ has kernel $\{0\}$ in $C(\mathcal{S})$; the same is true in $L^{2}(\mathcal{S})$ (see, e.g., [11, Prop. 3.13]) and then also in $L^{p}(\mathcal{S}), p \geq 2$. Thus, from Fredholm theory, $\frac{1}{2}+T$ has a bounded inverse on $L^{p}(\mathcal{S})$ for $2 \leq p<\infty$, and (4.1) has a unique solution $f \in L^{p}$ for each $g \in L^{p}$, with $\|f\|_{p} \leq C\|g\|_{p}$. We will argue that the discrete problem can be solved by regarding it as a perturbation of the exact problem, making a sequence of steps from (4.1) to (1.13). This is the stability part of the proof; the consistency part has been done in sections 2 and 3. We always assume (1.15). When we say a discrete operator is bounded, we mean bounded uniformly with respect to $h$.

4.1. The smoothing. The regularized kernel $K_{\delta}$ has the form $K_{\delta}\left(x, x^{\prime}\right)=$ $K\left(x, x^{\prime}\right) s\left(\left|x-x^{\prime}\right| / \delta\right)$, where $s(x) \rightarrow 1, D s(x) \rightarrow 0$ rapidly as $x \rightarrow \infty$. Using the smoothness of $K_{\delta}$ and $s$, we note the pointwise estimates

$$
\begin{aligned}
\left|K_{\delta}\left(x, x^{\prime}\right)\right| \leq C r^{-1}, & r \geq \delta ; & & \left|K_{\delta}\left(x, x^{\prime}\right)\right| \leq C \delta^{-1}, \quad r \leq \delta ; \\
\left|D K_{\delta}\left(x, x^{\prime}\right)\right| \leq C r^{-2}, & r \geq \delta ; & & \left|D K_{\delta}\left(x, x^{\prime}\right)\right| \leq C \delta^{-2}, \quad r \leq \delta,
\end{aligned}
$$

where $r=\left|x-x^{\prime}\right|$ and $D$ denotes an $\alpha$-derivative, with either $x=X^{\sigma}(\alpha)$ or $x^{\prime}=$ $X^{\sigma}(\alpha)$. We assume here that $x, x^{\prime}$ are in a bounded set such as $\mathcal{S}$.

To see the effect of the smoothing, we can estimate, as in section 2 ,

$$
\int_{S}\left|K_{\delta}\left(x, x^{\prime}\right)-K\left(x, x^{\prime}\right)\right| d S \leq C_{1} \int_{0}^{\infty} r^{-1}|s(r / \delta)-1| r d r=C_{2} \delta
$$

uniformly for $x \in S$; the same holds with $x, x^{\prime}$ reversed. In general, for any integral operator $\mathcal{A}$ on $\mathcal{S}$ with kernel $\mathcal{K}$, if we have uniform estimates

$$
\int_{\mathcal{S}}\left|\mathcal{K}\left(x, x^{\prime}\right)\right| d S\left(x^{\prime}\right) \leq M, \quad \int_{\mathcal{S}}\left|\mathcal{K}\left(x, x^{\prime}\right)\right| d S(x) \leq M,
$$

it follows that $\mathcal{A}$ is a bounded operator on $L^{p}(\mathcal{S})$ with $\|\mathcal{A}\| \leq M$ (see, e.g., [11, Prop. $0.10])$. In our case we conclude that the operator on $L^{p}(\mathcal{S})$ with kernel $K_{\delta}-K$ has norm $O(\delta)$. Thus, since the operator $\frac{1}{2}+T$ is invertible, the same is true for $\frac{1}{2}+T_{\delta}$, for $\delta$ small enough, where $T_{\delta}$ is the operator as in (4.1) with $K_{\delta}$ in place of $K$.

4.2. A discretized kernel. Next we modify the kernel $K_{\delta}$ so that it acts in a natural way on discrete functions. We work with grid functions $f_{i}^{\sigma}$, defined at each grid point $x_{i}^{\sigma}=X^{\sigma}(i h), i h \in V_{\sigma}$, for each $\sigma$. If $x_{i}^{\sigma}=x_{j}^{\tau}$ for some $i, j$ with $\sigma \neq \tau$, we require $f_{i}^{\sigma}=f_{j}^{\tau}$; this property is preserved by the discrete operators. Let $Q_{i}$ be the grid square in the $\alpha$-plane $Q_{i}=\left\{\alpha=\left(\alpha_{1}, \alpha_{2}\right):-h / 2<\alpha_{\nu}-i_{\nu} h \leq h / 2, \nu=1,2\right\}$, and let $\chi_{i}^{\sigma}(x)=1$ for $x=X^{\sigma}(\alpha)$, with $\alpha \in Q_{i}$, and $\chi_{i}^{\sigma}(x)=0$ otherwise; i.e., $\chi_{i}^{\sigma}$ is the characteristic function of the set $X^{\sigma}\left(Q_{i}\right)$. Given any grid function $f_{i}^{\sigma}$, we can associate a function $B f$ on $\mathcal{S}$ defined by

$$
(B f)(x)=\sum_{\sigma, i} \psi_{i}^{\sigma} \chi_{i}^{\sigma}(x) f_{i}^{\sigma} .
$$


The part of $B f$ from one $\sigma$ is piecewise constant, but $B f$ is not, since the parts overlap. Recalling (1.14), we define a modified kernel $\tilde{K}$ and operator $\tilde{T}$ on $L^{p}(\mathcal{S})$ as

$$
\begin{gathered}
\tilde{K}\left(x, x^{\prime}\right)=\sum_{i, \sigma, j, \tau} K_{i j}^{\sigma \tau} \psi_{i}^{\sigma} \psi_{j}^{\tau} \chi_{i}^{\sigma}(x) \chi_{j}^{\tau}\left(x^{\prime}\right) A_{j}^{\tau}=B\left(\sum_{j, \tau} K_{i j}^{\sigma \tau} \psi_{j}^{\tau} \chi_{j}^{\tau}\left(x^{\prime}\right) A_{j}^{\tau}\right), \\
\tilde{T} f(x)=\sum_{\tau} \int \tilde{K}\left(x, X^{\tau}(\alpha)\right) f\left(X^{\tau}(\alpha)\right) d \alpha .
\end{gathered}
$$

We write $K_{\delta}\left(x, x^{\prime}\right)$ as $\sum_{\sigma, \tau} K_{\delta}\left(x, x^{\prime}\right) \psi^{\sigma}(x) \psi^{\tau}\left(x^{\prime}\right)$; for each $\sigma, \tau, i, j$ we have replaced $K^{\sigma \tau} \equiv K_{\delta} \psi^{\sigma} \psi^{\tau} A^{\tau}$ on $X^{\sigma}\left(Q_{i}\right) \times X^{\tau}\left(Q_{j}\right)$ with the constant $\tilde{K}^{\sigma \tau} \equiv K_{\delta}\left(x_{i}^{\sigma}, x_{j}^{\tau}\right) \psi_{i}^{\sigma} \psi_{j}^{\tau} A_{j}^{\tau}$. Then for each $\sigma, \tau$ and each $x \in X^{\sigma}\left(U^{\sigma}\right)$ we estimate

$$
\int_{U_{\tau}}\left|\tilde{K}^{\sigma \tau}\left(x, X^{\tau}(\alpha)\right)-K^{\sigma \tau}\left(x, X^{\tau}(\alpha)\right)\right| d \alpha \leq C h \sum_{j} M_{i j}^{\sigma \tau} h^{2},
$$

where $i$ is such that $x \in X^{\sigma}\left(Q_{i}\right)$. Here $M_{i j}^{\sigma \tau}$ is a bound for $\left|D K^{\sigma \tau}\right|$ within radius $O(h)$ of $\left(x_{i}^{\sigma}, x_{j}^{\tau}\right)$. Using (4.4) we have $\left|M_{i j}^{\sigma \tau}\right| \leq C\left|x_{i}^{\sigma}-x_{j}^{\tau}\right|^{-2}$ for $\left|x_{i}^{\sigma}-x_{j}^{\tau}\right|>C_{0} \delta$ and $\left|M_{i j}^{\sigma \tau}\right| \leq C \delta^{-2}$ otherwise. Since $c_{1}\left|\alpha-\alpha^{\prime}\right| \leq\left|X^{\tau}(\alpha)-X^{\tau}\left(\alpha^{\prime}\right)\right| \leq c_{2}\left|\alpha-\alpha^{\prime}\right|$, we can bound (4.9) as follows, replacing the sum for $\left|x_{i}^{\sigma}-x_{j}^{\tau}\right| \geq O(\delta)$ by an $\alpha$-integral:

$$
C_{1} h \delta^{-2}(\delta / h)^{2} h^{2}+C_{2} h \int_{C_{3} \delta}^{C_{4}} r^{-2} r d r \leq C_{5} h(1+|\log \delta|) \leq C_{6} h(1+|\log h|)
$$

(cf. [5, Lemma 3.2] or [16, Lemma 5] for arguments of this type). This gives the first of two estimates of form (4.5), with $\mathcal{K}=\tilde{K}-K_{\delta}$, and the second is similar. Thus we can conclude that the norm of $\tilde{T}-T$, as an operator on $L^{p}(\mathcal{S})$, is $O(h|\log h|)$. Since the operator $\frac{1}{2}+T_{\delta}$ is invertible, the nearby operator $\frac{1}{2}+\tilde{T}$ is also invertible.

4.3. Discrete functions. Next we pass from functions of continuous $x \in \mathcal{S}$ to discrete functions. For a grid function $f_{i}^{\sigma}$ we use the $L_{h}^{p}$ norm, defined by

$$
\|f\|_{L_{h}^{p}}^{p}=\sum_{\sigma, i}\left|f_{i}^{\sigma}\right|^{p} h^{2}
$$

The operator $B$ of (4.6) is bounded from $L_{h}^{p}$ to $L^{p}$. Now, given a grid function $g \in L_{h}^{p}$, we have $B g \in L^{p}(\mathcal{S})$, and from the result above there is a unique $F \in L^{p}(\mathcal{S})$ depending boundedly on $B g \in L^{p}(S)$ so that $\frac{1}{2} F+\tilde{T} F=B g$, or, in view of (4.7), $F=-2 B w+2 B g$, where

$$
w_{i}^{\sigma}=\sum_{j, \tau} K_{i j}^{\sigma \tau} \psi_{j}^{\tau} A_{j}^{\tau} \int_{Q_{j}} F\left(X^{\tau}(\alpha)\right) d \alpha .
$$

Now we can define a discrete function $f$ so that $F=B f$ :

$$
f_{i}^{\sigma}=-2 w_{i}^{\sigma}+2 g_{i}^{\sigma} .
$$

This can be rewritten as

$$
\frac{1}{2} f_{i}^{\sigma}+\left(T_{h}(\mathcal{M} f)\right)_{i}^{\sigma}=g_{i}^{\sigma}, \quad(\mathcal{M} f)_{j}^{\tau}=h^{-2} \int_{Q_{j}} F\left(X^{\tau}(\alpha)\right) d \alpha, \quad F=B f
$$


where $T_{h}$ is the discrete integral operator from $L_{h}^{p}$ to $L_{h}^{p}$ defined by

$$
\left(T_{h} \bar{f}\right)_{i}^{\sigma}=\sum_{j, \tau} K_{i j}^{\sigma \tau} \psi_{j}^{\tau} \bar{f}_{j}^{\tau} A_{j}^{\tau} h^{2} .
$$

Equation (4.14) resembles the desired discrete integral equation (1.13) but has $(\mathcal{M} f)_{j}^{\tau}$ rather than $f_{j}^{\tau}$ inside the integral operator. It is easy to check that the mapping $F \mapsto \mathcal{M f}$ is bounded from $L^{p}$ to $L_{h}^{p}$, and thus $f \mapsto B f=F \mapsto \mathcal{M} f$ is bounded from $L_{h}^{p}$ to $L_{h}^{p}$. We can see that the discrete operator $T_{h}$ is bounded on $L_{h}^{p}$ using the pointwise estimate (4.3) and an argument similar to that for $\tilde{K}-K$ in (4.9), (4.10). We can now check that the discrete solution $f$ of (4.14) depends boundedly on $g$ : Given $g \in L_{h}^{p}, F \in L^{p}$, as determined by the equation $\left(\frac{1}{2}+\tilde{T}\right) F=B g$, depends boundedly on $B g \in L^{p}$ and therefore on $g \in L_{h}^{p}$. Then, using the boundedness of $T_{h}$, the discrete function $f$, defined by (4.12), (4.13), depends boundedly in $L_{h}^{p}$ on $g$. The solution $f$ of (4.14) is unique since the corresponding solution $F$ of $\left(\frac{1}{2}+\tilde{T}\right) F=B g$ is unique. We have shown that the operator $\mathcal{A}$ has a bounded inverse on $L_{h}^{p}$, where

$$
\mathcal{A} f=\frac{1}{2} f+T_{h} \mathcal{M} f \text {. }
$$

4.4. Boundedness in the Hölder norm. We will now improve the solvability estimate for $\mathcal{A}$ to a higher norm in order to measure the agreement of values on overlapping grids. For $0<\lambda<1$, we define the discrete Hölder norm as

$$
\|f\|_{C_{h}^{\lambda}}=\sup _{\sigma, i}\left|f_{i}^{\sigma}\right|+\sup _{\sigma, i, \sigma^{\prime}, i^{\prime}}\left|f_{i}^{\sigma}-f_{i^{\prime}}^{\sigma^{\prime}}\right| /\left|x_{i}^{\sigma}-x_{i^{\prime}}^{\sigma^{\prime}}\right|^{\lambda},
$$

excluding pairs with $x_{i}^{\sigma}=x_{i^{\prime}}^{\sigma^{\prime}}$ in the latter sup. Similarly, $C_{h}^{0}$ will be the space with the supremum norm. We first consider the operator $\mathcal{A}$ on $C_{h}^{0}$. From (4.3) we see that, for $q<2, \sum_{j}\left|K_{i j}^{\sigma \tau}\right|^{q} h^{2} \leq C$. Then, from Hölder's inequality, $T_{h}$ is bounded from $L_{h}^{p}$ to $C_{h}^{0}$, provided $p>2$. Now if $\frac{1}{2} f+T_{h} \mathcal{M} f=g$, we have $\left\|T_{h} \mathcal{M} f\right\|_{C_{h}^{0}} \leq C_{1}\|g\|_{L_{h}^{p}} \leq$ $C_{2}\|g\|_{C_{h}^{0}}$, and so $\|f\|_{C_{h}^{0}} \leq C_{3}\|g\|_{C_{h}^{0}}$. Thus $\mathcal{A}$ has a bounded inverse on $C_{h}^{0}$.

Next we check that $T_{h}$ is bounded from $C_{h}^{0}$ to $C_{h}^{\lambda}$. For arbitrary grid points $x_{i}^{\sigma}$ and $x_{i^{\prime}}^{\sigma^{\prime}}$, let $d=\left|x_{i}^{\sigma}-x_{i^{\prime}}^{\sigma^{\prime}}\right|$. We need to verify that (with $\tau$ fixed)

$$
d^{-\lambda} \sum_{j}\left[K_{i j}^{\sigma \tau}-K_{i^{\prime} j}^{\sigma^{\prime} \tau}\right] \psi_{j}^{\tau} f_{j}^{\tau} A_{j}^{\tau} h^{2}
$$

is bounded, assuming $f$ bounded in $C_{0}^{h}$. We use the estimates (4.3), (4.4) to bound

$$
K_{i j}^{\sigma \tau}-K_{i^{\prime} j}^{\sigma^{\prime} \tau}=K_{\delta}\left(x_{i}^{\sigma}, x_{j}^{\tau}\right)-K_{\delta}\left(x_{i^{\prime}}^{\sigma^{\prime}}, x_{j}^{\tau}\right) .
$$

As in classical arguments, we split the sum into two parts. For $j$ in the set $J_{1}$ such that $\left|x_{i}^{\sigma}-x_{j}^{\tau}\right| \leq 2 d$, we apply (4.3) to the two terms separately. If $d<\delta$ we have

$$
\sum_{j \in J_{1}}\left|K_{i j}^{\sigma \tau}\right| h^{2} \leq C_{1} \delta^{-1}(d / h)^{2} h^{2} \leq C_{2} d
$$

and similarly for $K_{i^{\prime} j}^{\sigma^{\prime} \tau}$, whereas, for larger $d$, the sum is bounded by

$$
C_{3} \delta^{-1}(\delta / h)^{2} h^{2}+C_{4} \int_{C_{5} \delta}^{C_{6} d} r^{-1} r d r \leq C_{7} \delta+C_{8} d \leq C_{9} d .
$$


For the remaining sum over the set $J_{2}$ with $\left|x_{i}^{\sigma}-x_{j}^{\tau}\right|>2 d$, we bound (4.19) by $d$ times $D K\left(z, x_{j}^{\tau}\right)$, with $z$ along the line from $x_{i}^{\sigma}$ to $x_{i^{\prime}}^{\sigma^{\prime}}$. With $r=\left|x_{j}^{\tau}-x_{i}^{\sigma}\right|$, we have $\left|x_{j}^{\tau}-z\right| \geq r-d \geq r / 2$. Thus (4.19) is bounded by $C d r^{-2}$ for $r>\delta$ or $C d \delta^{-2}$ otherwise, using (4.4). Now if $d \geq \delta$ we get

$$
\sum_{j \in J_{2}}\left|K_{i j}^{\sigma \tau}-K_{i^{\prime} j}^{\sigma^{\prime} \tau}\right| h^{2} \leq C_{1} d \int_{C_{2} d}^{C_{3}} r^{-2} r d r \leq C_{4} d(1+|\log d|),
$$

and otherwise we have this plus an additional term $C_{5} d \delta^{-2}(\delta / h)^{2} h^{2} \leq C_{6} d$. We conclude that the Hölder quotient (4.18) is bounded by

$$
C_{1} d^{1-\lambda}(|\log d|+1)\|f\|_{C_{h}^{0}} \leq C_{2}\|f\|_{C_{h}^{0}},
$$

and, consequently, for any $f \in C_{h}^{0},\left\|T_{h} f\right\|_{C_{h}^{\lambda}} \leq C_{3}\|f\|_{C_{h}^{0}}$ as claimed.

We have already shown that the operator $\mathcal{A}$ has a bounded inverse on $C_{h}^{0}$; we can now show the same is true for $C_{h}^{\lambda}$. If $\frac{1}{2} f+T_{h} \mathcal{M} f=g$ with $g \in C_{h}^{\lambda}$, then $\|\mathcal{M} f\|_{C_{h}^{0}} \leq C_{1}\|f\|_{C_{h}^{0}} \leq C_{2}\|g\|_{C_{h}^{0}}$, and thus $\left\|T_{h} \mathcal{M} f\right\|_{C_{h}^{\lambda}} \leq C_{3}\|g\|_{C_{h}^{0}} \leq C_{3}\|g\|_{C_{h}^{\lambda}}$. Since $f=2 g-2 T_{h} \mathcal{M} f$, it follows that $\|f\|_{C_{h}^{\lambda}} \leq C\|g\|_{C_{h}^{\lambda}}$.

4.5. Correcting the discrete equation. Next, in order to compare the operator $T_{h} \mathcal{M}$ with $T_{h}$, we show that $\mathcal{M} f$, as defined in (4.14), is close to $f$ in $C_{h}^{0}$, relative to the $C_{h}^{\lambda}$-norm of $f$, for small $h$. Given a grid function $f$ and $x \in \mathcal{S}$, suppose $x \in X^{\tau}\left(Q_{j}\right)$ for some $\tau, j$ so that $\left|x-x_{j}^{\tau}\right| \leq C h$. If also $x \in X^{\sigma}\left(Q_{i}\right)$, then $\left|x-x_{i}^{\sigma}\right| \leq C h$ so that $\left|x_{i}^{\sigma}-x_{j}^{\tau}\right| \leq 2 C h$. Thus $\left|f_{i}^{\sigma}-f_{j}^{\tau}\right| \leq C_{1} h^{\lambda}\|f\|_{C_{h}^{\lambda}}$. Similarly, $\psi_{i}^{\sigma}-\psi^{\sigma}(x)=O(h)$. Applying this to the definition of $B f$, we find $\left|(B f)(x)-f_{j}^{\tau}\right| \leq C_{2} h^{\lambda}\|f\|_{C_{h}^{\lambda}}$ uniformly for $x \in X^{\tau}\left(Q_{j}\right)$. Then since $(\mathcal{M} f)_{j}^{\tau}$ is an average of $B f$ over this set,

$$
\left|(\mathcal{M} f)_{j}^{\tau}-f_{j}^{\tau}\right| \leq C_{2} h^{\lambda}\|f\|_{C_{h}^{\lambda}} .
$$

The last result has an important consequence: Since $\mathcal{M}$ is close to the identity as an operator from $C_{h}^{\lambda}$ to $C_{h}^{0}$, and since $T_{h}$ is bounded from $C_{h}^{0}$ to $C_{h}^{\lambda}$, the operators $f \mapsto \frac{1}{2} f+T_{h} \mathcal{M} f$ and $f \mapsto \frac{1}{2} f+T_{h} f$, acting on $C_{h}^{\lambda}$, are close when $h$ is small. Since the first has a bounded inverse on $C_{h}^{\lambda}$, the second does as well. That is, we have a bounded solution operator on $C_{h}^{\lambda}$ for the equation $\frac{1}{2} f+T_{h} f=g$. This equation is the analogue of (1.13) without the subtracted term.

4.6. The subtraction. Now let $z_{i}^{\sigma}=T_{h} \cdot 1$. The sum $z_{i}^{\sigma}$ approximates an integral which, according to (1.7), is identically $\frac{1}{2}$. We check that $\left\|z_{i}^{\sigma}-\frac{1}{2}\right\|_{C_{h}^{\lambda}} \rightarrow 0$ as $h, \delta \rightarrow 0$ : The estimates of sections 2 and 3 show that $\left|z_{i}^{\sigma}-\frac{1}{2}\right| \leq C(h+\delta)$ uniformly in $i, \sigma$. As for the Hölder quotient, we see from (4.20) that it is small for $d$ small, say, $d \leq d_{0}$, with $d_{0}$ independent of $h$. But for $d \geq d_{0}$ it is bounded by $C h d_{0}^{-\lambda}$, which is small when $h$ is small enough. As a consequence, the operators $f \mapsto \frac{1}{2} f+T_{h} f$ and $f \mapsto f+T_{h} f-z f$ on $C_{h}^{\lambda}$ are close for $h$ small. As before, we conclude that the new operator has a bounded inverse. This is exactly the statement that the discrete integral equation (1.13) is solvable, with solution bounded in $C_{h}^{\lambda}$, that is, $\|f\|_{C_{h}^{\lambda}} \leq C\|g\|_{C_{h}^{\lambda}}$.

We wish to show that the solution of (1.13) is also bounded on $C_{h}^{0}$, since this norm is more convenient for comparing with the exact solution. We can write the equation as $(1-z) f+T_{h} f=g$ or $f+\zeta T_{h} f=\zeta g$, where $\zeta=1 /(1-z)$. Since $z \approx \frac{1}{2}$ in $C_{h}^{\lambda}$, $\zeta \approx 2$ in $C_{h}^{\lambda}$. Rearranging the terms, we have $(1-z)(f-\zeta g)+T_{h}(f-\zeta g)=-T_{h}(\zeta g)$. 
Now, using our last result, with $f-\zeta g$ in place of $f$, and the bound for $T_{h}: C_{h}^{0} \rightarrow C_{h}^{\lambda}$, we find $\|f-\zeta g\|_{C_{h}^{\lambda}} \leq C_{1}\left\|T_{h}(\zeta g)\right\|_{C_{h}^{\lambda}} \leq C_{2}\|g\|_{C_{h}^{0}}$ from which it follows that

$$
\|f\|_{C_{h}^{0}} \leq C\|g\|_{C_{h}^{0}}, \quad f=\left((1-z) I+T_{h}\right)^{-1} g .
$$

4.7. The error estimate. Having established the bounded solvability (4.22) for the discrete integral equation (1.13), we can easily estimate the error when the exact solution is smooth. Suppose $f, g$ are smooth functions on $\mathcal{S}$ satisfying (4.1). (If $g$ is smooth, it follows that $f$ is smooth.) Let $g_{i}^{\sigma}$ be the grid function taking values $g_{i}^{\sigma}=g\left(x_{i}^{\sigma}\right)$, and let $f_{i}^{\sigma}$ be the solution of (1.13). Define $e_{i}^{\sigma}=f_{i}^{\sigma}-f\left(x_{i}^{\sigma}\right)$. Subtracting, we get the error equation

$$
\begin{gathered}
e_{i}^{\sigma}+\sum_{j, \tau} K_{i j}^{\sigma \tau} \psi_{j}^{\tau}\left[e_{j}^{\tau}-e_{i}^{\sigma}\right] A_{j}^{\tau} h^{2}+r_{i}^{\sigma}, \\
r_{i}^{\sigma}=\int K\left(x_{i}^{\sigma}, x^{\prime}\right)\left[f\left(x^{\prime}\right)-f\left(x_{i}^{\sigma}\right)\right] d x^{\prime}-\sum_{j, \tau} K_{i j}^{\sigma \tau} \psi_{j}^{\tau}\left[f\left(x_{j}^{\tau}\right)-f\left(x_{i}^{\sigma}\right)\right] A_{j}^{\tau} h^{2} .
\end{gathered}
$$

The $O\left(\delta^{5}\right)$ smoothing estimate of section 2 and the discretization estimate (3.25) apply to $r_{i}^{\sigma}$, since $f$ is smooth, and we have $\left|r_{i}^{\sigma}\right| \leq \varepsilon(h, \delta)$, uniformly in $i, \sigma$, where $\varepsilon(h, \delta)$ is the right side of (1.17). A similar estimate follows for $e_{i}^{\sigma}$, after applying (4.22) to the error equation (4.23), and we have $\left|f_{i}^{\sigma}-f\left(x_{i}^{\sigma}\right)\right| \leq \varepsilon(h, \delta)$. Thus we have proved the error estimate (1.17) of Theorem 1.1.

4.8. The iteration. Finally, we discuss the convergence of the iterates (1.16) to the solution of the discrete integral equation (1.13). It is a fact of potential theory that the operator $T$ has spectrum in the interval $-\frac{1}{2}<\lambda \leq \frac{1}{2}$ (see, e.g., [18, section 10.5] or [9, section 5.1]). Then, for $0<\beta<1$, the related operator $(1-\beta) I-2 \beta T$ has spectral radius $<1$, and the convergence of (1.5) follows. For the discrete case we can argue that the approximations to $T$ above perturb this spectral radius only slightly, and thus the discrete iteration (1.16) also converges. We omit the details.

5. Numerical examples. We present the results of three test problems illustrating the solution of the integral equation and the subsequent calculation of the solution of the Dirichlet problem (1.1) at points near the surface. The results generally verify the predictions of the theory.

For our first two examples the surface $\mathcal{S}$ is the unit sphere $x_{1}^{2}+x_{2}^{2}+x_{3}^{2}=1$. We cover the sphere with two stereographic projections. The projection on the equatorial plane by rays through the south pole $(0,0,-1)$ gives the first coordinate system, $X^{1}: \mathbf{R}^{2} \rightarrow U^{1}=S-\{(0,0,-1)\}:$

$$
x_{1}=\frac{2 \alpha_{1}}{1+|\alpha|^{2}}, \quad x_{2}=\frac{2 \alpha_{2}}{1+|\alpha|^{2}}, \quad x_{3}=\frac{1-|\alpha|^{2}}{1+|\alpha|^{2}} .
$$

Similarly, projecting from the north pole gives a second system $X^{2}: \mathbf{R}^{2} \rightarrow U^{2}=S-$ $\{(0,0,1)\}$ as in (5.1) but with $x_{3} \rightarrow-x_{3}$. Each system maps the unit disk in the plane to a hemisphere; we use disks of radius 1.25 so that the two systems overlap. To define the partition of unity $\left\{\psi^{1}, \psi^{2}\right\}$, we first set $\phi^{\sigma}\left(X^{\sigma}(\alpha)\right)=\exp \left(-1.25^{2} /\left(1.25^{2}-|\alpha|^{2}\right)\right)$ for $|\alpha| \leq 1.25$ and $\phi^{\sigma}=0$ otherwise; then $\phi^{\sigma}$ is a smooth function with support $\left\{X^{\sigma}(\alpha):|\alpha| \leq 1.25\right\}$. We then define $\psi^{\sigma}(x)=\phi^{\sigma}(x) /\left(\phi^{1}(x)+\phi^{2}(x)\right)$. Grid points $\alpha_{i}=i$ are introduced with $X^{\sigma}\left(\alpha_{i}\right) \in V^{\sigma}$, where $V^{\sigma}$ is the interior of the support of $\psi^{\sigma}$. These sets are $V^{1}=\mathcal{S} \cap\left\{x_{3}>-9 / 41\right\}, V^{2}=\mathcal{S} \cap\left\{x_{3}<9 / 41\right\}$, corresponding 
TABLE 1

The integral equation with third order kernel.

\begin{tabular}{|rr||rcc||rrr||rr|}
\hline \multicolumn{1}{|c||}{} & \multicolumn{3}{c||}{$\delta=.5 h^{2 / 3}$} & \multicolumn{3}{c||}{$\delta=.75 h^{2 / 3}$} & \multicolumn{2}{c|}{$\delta=2 h$} \\
$1 / h$ & Grid pts & $\delta / h$ & Rel err & Order & $\delta / h$ & Rel err & Order & Rel err & Order \\
\hline \hline 8 & 610 & 1.00 & $1.7 \mathrm{E}-3$ & & 1.50 & $6.0 \mathrm{E}-3$ & & $1.4 \mathrm{E}-2$ & \\
16 & 2490 & 1.26 & $4.8 \mathrm{E}-4$ & 1.9 & 1.89 & $1.6 \mathrm{E}-3$ & 1.9 & $1.9 \mathrm{E}-3$ & 2.9 \\
32 & 10026 & 1.59 & $1.2 \mathrm{E}-4$ & 2.0 & 2.38 & $4.0 \mathrm{E}-4$ & 2.0 & $2.4 \mathrm{E}-4$ & 3.0 \\
\hline
\end{tabular}

TABLE 2

The integral equation with fifth order kernel.

\begin{tabular}{|rr||rcc||ccc||cr|}
\hline \multicolumn{1}{|c||}{} & \multicolumn{3}{c||}{$\delta=.5 h^{2 / 3}$} & \multicolumn{3}{c||}{$\delta=.75 h^{2 / 3}$} & \multicolumn{2}{c|}{$\delta=2 h$} \\
$1 / h$ & Grid pts & $\delta / h$ & Rel err & Order & $\delta / h$ & Rel err & Order & Rel err & Order \\
\hline \hline 8 & 610 & 1.00 & $6.0 \mathrm{E}-4$ & & 1.50 & $5.9 \mathrm{E}-4$ & & $4.8 \mathrm{E}-4$ & \\
16 & 2490 & 1.26 & $9.5 \mathrm{E}-5$ & 2.7 & 1.89 & $1.5 \mathrm{E}-5$ & 5.3 & $2.0 \mathrm{E}-5$ & 4.6 \\
32 & 10026 & 1.59 & $7.7 \mathrm{E}-6$ & 3.6 & 2.38 & $1.7 \mathrm{E}-6$ & 3.1 & $9.0 \mathrm{E}-7$ & 4.5 \\
64 & 40138 & 2.00 & $1.4 \mathrm{E}-7$ & 5.7 & 3.00 & $1.7 \mathrm{E}-7$ & 3.3 & $1.4 \mathrm{E}-7$ & 2.6 \\
\hline
\end{tabular}

to $|\alpha|<1.25$. The metric tensor in each patch is $g_{i j}=4\left(|\alpha|^{2}+1\right)^{-2} \delta_{i j}$, and the area factor $A=\sqrt{g}$ is $4\left(|\alpha|^{2}+1\right)^{-2}$. The surface Laplacian is $\frac{1}{4}\left(|\alpha|^{2}+1\right)^{2}\left(\partial_{11}+\partial_{22}\right)$. These quantities could be computed from the points $\left\{X^{\sigma}\left(\alpha_{i}\right)\right\}$, but we used analytical values in our computations.

Our first example is based on a spherical harmonic, so that the solution for the integral equation is known, as well as for the boundary value problem. We define

$$
f(x)=1.75\left(y_{1}-2 y_{2}\right)\left(7.5 y_{3}^{2}-1.5\right), \quad y=M x,
$$

with $M=(1 / \sqrt{6})\left(\sqrt{2}(1,1,1)^{T}, \sqrt{3}(0,1,-1)^{T},(-2,1,1)^{T}\right)$, an orthogonal matrix. We use $M$ to avoid rectangular symmetry in the test problem. The functions $f(x / r) r^{3}$ and $f(x / r) / r^{4}, r=|x|$, are both harmonic. The double layer potential $u$ due to $f$ is determined by the jump in $u$ and the fact that $\partial u / \partial n$ is continuous. It is

$$
u(x)=(4 / 7) f(x / r) r^{3}, \quad r<1 ; \quad u(x)=(-3 / 7) f(x / r) / r^{4}, \quad r>1 .
$$

Thus if we set $g(x)=(4 / 7) f(x)$ on $\mathcal{S}$, the solution of the Dirichlet problem (1.1) is $u$, as defined above for $r<1$, and the solution of the integral equation (1.4) is $f$.

We solved the discrete form (1.13) of the exact integral equation (1.4) using 12 iterations of (1.16) with $\beta=.7$. We tested the third order kernel (1.10) as well as the fifth order one (1.12) to check the order of accuracy. The results are reported in Tables 1 and 2. A grid size $h$ in the coordinate systems and the total number of grid points are displayed. Each coordinate patch is a disk with $5 / 2 h$ points along a diameter. We choose the smoothing radius $\delta$ proportional to $h^{q}, q=2 / 3$ or 1 . The relative error displayed is the maximum error divided by $\max _{\mathcal{S}} f \approx 8.1$. The computed order of accuracy is found from two successive cases. With the third order kernel, the expected order of accuracy $3 q$ is clearly visible in Table 1 . The errors shown in Table 2 for the fifth order kernel are much smaller. With $q=2 / 3$ the predicted order $10 / 3$ is less evident, but it appears to take over in the second case, with the larger $\delta$. With $q=1$, $\delta=2 h$, the order is at first near 5 but deteriorates as $h$ is decreased, as we should expect from the analysis.

After solving the integral equation with a choice of $h$ and $\delta$, using the fifth order kernel, we then computed the solution $u$ at points near $\mathcal{S}$ with the same $h$ and $\delta$. To select a set of points, we cover $\mathbf{R}^{3}$ with a $3 \mathrm{D}$ mesh of spacing $h$; it is an arbitrary 
TABLE 3

Nearby points for the first problem.

\begin{tabular}{|rr||rr||rr||rr|}
\hline \multicolumn{1}{|c||}{} & \multicolumn{2}{|c||}{ Irreg } & \multicolumn{2}{c||}{$\delta=.5 h^{2 / 3}$} & \multicolumn{2}{c|}{$\delta=.75 h^{2 / 3}$} & \multicolumn{2}{c|}{$\delta=2 h$} \\
$1 / h$ & points & Rel err & Order & Rel err & Order & Rel err & Order \\
\hline \hline 8 & 606 & $1.2 \mathrm{E}-2$ & & $1.4 \mathrm{E}-2$ & & $2.1 \mathrm{E}-2$ & \\
16 & 2546 & $6.2 \mathrm{E}-4$ & 4.3 & $1.9 \mathrm{E}-3$ & 2.9 & $2.3 \mathrm{E}-3$ & 3.2 \\
32 & 10470 & $1.4 \mathrm{E}-4$ & 2.1 & $4.7 \mathrm{E}-4$ & 2.0 & $2.8 \mathrm{E}-4$ & 3.0 \\
64 & 42282 & $3.5 \mathrm{E}-5$ & 2.0 & $1.1 \mathrm{E}-4$ & 2.1 & $3.5 \mathrm{E}-5$ & 3.0 \\
\hline
\end{tabular}

TABLE 4

Nearby points for the second problem.

\begin{tabular}{|rr||rr||rr||rr|}
\hline \multicolumn{1}{|c||}{ Irreg } & \multicolumn{2}{c||}{$\delta=.5 h^{2 / 3}$} & \multicolumn{2}{c|}{$\delta=.75 h^{2 / 3}$} & \multicolumn{2}{c|}{$\delta=2 h$} \\
$1 / h$ & points & Rel err & Order & Rel err & Order & Rel err & Order \\
\hline \hline 8 & 606 & $1.6 \mathrm{E}-2$ & & $3.2 \mathrm{E}-2$ & & $4.7 \mathrm{E}-2$ & \\
16 & 2546 & $4.2 \mathrm{E}-4$ & 5.3 & $1.3 \mathrm{E}-3$ & 4.6 & $1.6 \mathrm{E}-3$ & 4.9 \\
32 & 10470 & $1.1 \mathrm{E}-4$ & 1.9 & $3.6 \mathrm{E}-4$ & 1.9 & $2.2 \mathrm{E}-4$ & 2.9 \\
64 & 42282 & $2.7 \mathrm{E}-5$ & 2.0 & $8.8 \mathrm{E}-5$ & 2.0 & $2.7 \mathrm{E}-5$ & 3.0 \\
\hline
\end{tabular}

choice to use the same $h$ in $\mathbf{R}^{3}$ as on $\mathcal{S}$. We select the set of "irregular" points $\left(i_{1} h, i_{2} h, i_{3} h\right)$ inside $\mathcal{S}$ for which the stencil of the five-point discrete Laplacian crosses the surface; i.e., the two points obtained by displacement of $\pm h$ in some coordinate are on different sides of $\mathcal{S}$. All such points are within $h$ of $\mathcal{S}$, and, for $h$ small, all points within $c h$ of $\mathcal{S}$ have this property if $c<1 / \sqrt{3}$. Thus they provided a good sample of $3 \mathrm{D}$ grid points near $\mathcal{S}$. These are the interior points needed to form the discrete Laplacian of $u$ in order to recover the values elsewhere; cf. [21] or [3].

For each selected point $y$ we find the closest point $x_{0}$ on $\mathcal{S}$. We need $f$ and the first two derivatives at $x_{0}$ for the subtraction and corrections. We find these from the computed values of $f$ at the grid points by Lagrange interpolation. We compute the sum (1.19) and add the smoothing and discretization corrections (1.21)-(1.25). Table 3 gives the number of irregular points; the relative error, found as the maximum error divided by $\max _{\mathcal{S}} g \approx 4.6$; and the computed order of accuracy. With $\delta=c h^{q}$, $q=2 / 3$ or 1 , we see the expected order $3 q=2$ or 3 .

Our second test problem, still with the unit sphere, is the harmonic function

$$
u(x)=\exp \left(y_{1}+2 y_{2}\right) \cos \sqrt{5} y_{3}, \quad y=M x,
$$

with $M$ as before. We prescribe $g(x)=u(x)$ on $\mathcal{S}$ and solve the integral equation within an error tolerance, again with $\beta=.7$. In this case we do not know the solution $f$ of the integral equation; however, we use the computed $f$ to find $u$ at the irregular points, as before, and compare with the exact solution in (5.3). Results are reported in Table 4. Again the predicted order of accuracy is evident as $h$ decreases. The error displayed is the maximum error divided by $\max _{\mathcal{S}} g \approx 9.4$.

For our third problem the surface $\mathcal{S}$ is the ellipsoid $x_{1}^{2}+x_{2}^{2}+x_{3}^{2} / 2=1$. We use coordinate systems similar to those for the sphere, with $x_{3}$ in (5.1) multiplied by $\sqrt{2}$. As the test problem we take the same harmonic function $u$ in (5.3). The coordinate systems are symmetric, but the functions are not, because of the rotation by $M$. The needed geometric quantities can be found analytically in this case. The coordinates are not orthogonal, as they were for the sphere, so that the formulas are tested in a more general setting. We solve the integral equation as before. We then select the set of irregular points by the same criterion and compute the solution $u$ on this set as a double layer potential, with corrections, using the solution $f$ of the integral equation. 
TABLE 5

Nearby points for the ellipsoid.

\begin{tabular}{|rr||rr||rr||rr|}
\hline \multicolumn{1}{|c||}{} & \multicolumn{2}{|c||}{ Irreg } & \multicolumn{2}{c||}{$\delta=.5 h^{2 / 3}$} & \multicolumn{2}{c|}{$\delta=.75 h^{2 / 3}$} & \multicolumn{2}{c|}{$\delta=2 h$} \\
$1 / h$ & points & Rel err & Order & Rel err & Order & Rel err & Order \\
\hline \hline 8 & 798 & $1.7 \mathrm{E}-2$ & & $3.3 \mathrm{E}-2$ & & $4.7 \mathrm{E}-2$ & \\
16 & 3330 & $3.2 \mathrm{E}-4$ & 5.7 & $1.0 \mathrm{E}-3$ & 5.0 & $1.2 \mathrm{E}-3$ & 5.3 \\
32 & 13614 & $8.3 \mathrm{E}-5$ & 2.0 & $2.7 \mathrm{E}-4$ & 1.9 & $1.6 \mathrm{E}-4$ & 2.9 \\
64 & 54914 & $2.1 \mathrm{E}-5$ & 2.0 & $6.6 \mathrm{E}-5$ & 2.0 & $2.1 \mathrm{E}-5$ & 3.0 \\
\hline
\end{tabular}

TABLE 6

Nearby points with $h=1 / 32$, varying $\delta$.

\begin{tabular}{|l||r|r|r|r|r|r|r|}
\hline$\delta / h$ & .01 & .1 & .5 & 1 & 2 & 5 & 10 \\
\hline Rel err & $3.7 \mathrm{E}-4$ & $3.7 \mathrm{E}-4$ & $2.8 \mathrm{E}-4$ & $6.8 \mathrm{E}-5$ & $1.6 \mathrm{E}-4$ & $2.2 \mathrm{E}-3$ & $1.5 \mathrm{E}-2$ \\
\hline
\end{tabular}

For each irregular point $y$ we need to find the point $x_{0}$ on the surface so that $y$ is along the normal from $x_{0}$, as well as the distance; we do this using Newton's method. The results, displayed in Table 5, are similar to those for the sphere. Again the relative error is the maximum error divided by $\max _{\mathcal{S}} g \approx 9.4$.

For the last problem we show in Table 6 the results of computing the solution at the irregular points using various choices of the smoothing parameter $\delta$. We first solve the integral equation with $h=1 / 32$ and $\delta=2 h$. We then compute the values at the irregular points, chosen with $h=1 / 32$, for various values of $\delta / h$, to verify the effects of the two corrections. For small $\delta / h$, the discretization correction is dominant; when $\delta / h$ is extremely small, more terms are needed in the sum (1.25). For larger $\delta / h$ the smoothing correction is important, and the discretization correction is negligible. As $\delta / h$ increases, the remaining smoothing error becomes significant.

\section{REFERENCES}

[1] C. Anderson, A method of local corrections for computing the velocity field due to a distribution of vortex blobs, J. Comput. Phys., 62 (1986), pp. 111-123.

[2] K. E. AtKinson, The Numerical Solution of Integral Equations of the Second Kind, Cambridge University Press, Cambridge, UK, 1997.

[3] J. T. BeAle And M.-C. LAI, A method for computing nearly singular integrals, SIAM J. Numer. Anal., 38 (2001), pp. 1902-1925.

[4] J. T. Beale, A convergent boundary integral method for three-dimensional water waves, Math. Comp., 70 (2001), pp. 977-1029.

[5] J. T. Beale and A. Majda, Vortex methods, I: Convergence in three dimensions, Math. Comp., 39 (1982), pp. 1-27.

[6] J. T. BeAle AND A. MAJDA, High order accurate vortex methods with explicit velocity kernels, J. Comput. Phys., 58 (1985), pp. 188-208.

[7] O. Bruno and L. Kunyansky, A fast, high-order algorithm for the solution of surface scattering problems: Basic implementation, tests, and applications, J. Comput. Phys., 169 (2001), pp. 80-110.

[8] L. Canino, J. Ottusch, M. Stalzer, J. Visher, and S. Wandzura, Numerical solution of the Helmholtz equation in $2 D$ and $3 D$ using a high-order Nyström discretization, J. Comput. Phys., 146 (1998), pp. 627-663.

[9] D. Colton and R. Kress, Integral Equation Methods in Scattering Theory, Wiley, New York, 1983.

[10] R. CorTez, The method of regularized Stokeslets, SIAM J. Sci. Comput., 23 (2001), pp. 12041225.

[11] G. Folland, Introduction to Partial Differential Equations, Princeton University Press, Princeton, NJ, 1995.

[12] J. Goodman, T. Y. Hou, and J. Lowengrub, Convergence of the point vortex method for the 2-D Euler equations, Comm. Pure Appl. Math., 43 (1990), pp. 415-430. 
[13] I. Graham And I. Sloan, Fully discrete spectral boundary integral methods for Helmholtz problems on smooth closed surfaces in $R^{3}$, Numer. Math., 92 (2002), pp. 289-323.

[14] L. Greengard and V. Rokhlin, A new version of the fast multipole method for the Laplace equation in three dimensions, Acta Numer., 6 (1997), pp. 229-269.

[15] W. HackBusch, Integral Equations, Theory and Numerical Treatment, Birkhäuser, Basel, 1995.

[16] O. H. HALd, Convergence of vortex methods for Euler's equations. II, SIAM J. Numer. Anal., 16 (1979), pp. $726-755$.

[17] Q. Huang And T. A. CRuse, Some notes on singular integral techniques in boundary element analysis, Internat. J. Numer. Methods Engrg., 36 (1993), pp. 2643-2659.

[18] R. Kress, Linear Integral Equations, 2nd ed., Springer, New York, 1999.

[19] C. Lage And C. SchWAB, Wavelet Galerkin algorithms for boundary integral equations, SIAM J. Sci. Comput., 20 (1999), pp. 2195-2222.

[20] J. S. Lowengrub, M. J. Shelley, And B. Merriman, High-order and efficient methods for the vorticity formulation of the Euler equations, SIAM J. Sci. Comput., 14 (1993), pp. 11071142.

[21] A. MAYO, Fast high order accurate solution of Laplace's equation on irregular regions, SIAM J. Sci. Statist. Comput., 6 (1985), pp. 144-157.

[22] M. Nitsche, Axisymmetric vortex sheet motion: Accurate evaluation of the principal value integral, SIAM J. Sci. Comput., 21 (1999), pp. 1066-1084.

[23] A. RATHSFELD, Nyström's method and iterative solvers for the solution of the double-layer potential equation over polyhedral boundaries, SIAM J. Numer. Anal., 32 (1995), pp. 924951.

[24] A. Rathsfeld, Quadrature methods for $2 D$ and $3 D$ problems, J. Comput. Appl. Math., 125 (2000), pp. 439-460.

[25] C. Schwab and W. L. Wendland, On the extraction technique in boundary integral equations, Math. Comp., 68 (1999), pp. 91-122. 